\title{
VOLUME XXII
}

\section{INDEX OF PAPERS AND TECHNICAL NOTES BY AUTHORS}

\section{Short papers and notes are marked $(N)$ in this index}

Author

Title

Apelkrans, Mats Y. T. On Difference Schemes for Hyperbolic Equations with Discon-

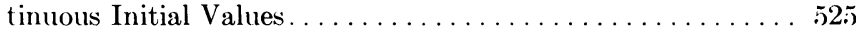

Atchison, T. A.

Atrin, A. O. L.

BAER, R. M. \& BRoCK, $\mathrm{P}$.

BANKS, D. O. \& Kurowski, G. J.

Bard, Yonathan

Bareiss, ErWin II.

Barnhill, Robert E.

Barnhill, Rober't E.

Bergland, G. D.

Bergman, S., Herriot, J. G. \& KURTZ, T. G.

Best, Gilber't C.

Bratley, Paul $\& \mathrm{MCK}_{\mathrm{AY}}$, JoHN

BROCK, $\mathrm{P}$.

Burgoyne, F. 1).

Buschman, R. G.

BYRD, P. F.

Campbell, C. M. \& $\mathrm{K}_{\mathrm{EAST}}, \mathrm{P}$.

ChaK, A. M.

Charmonman, S. \& Julius, R. S.

Chawla, M. M. \& J.IN, M. K.

Chawla, M. M. \& J AIN, M. K.

Chawla, M. M.

Chawla, M. M.

Cheema, M. S. \& Gupta, H.

Chiarella, C. \& Reichel, A.

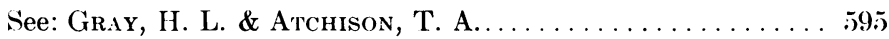

Explicit Gap Series at Cusps of $\Gamma(p)(\mathrm{N}) \ldots \ldots \ldots \ldots \ldots \ldots 416$

Natural Sorting Over Permutation Spaces............. 38

Computation of Eigenvalues of Singular Sturm-Liouville Sys-

tems................................... 304

On a Numerical Instability of Davidon-Like Methods (N)... . 665

Sylvester's Identity and Multistep Integer-Preserving Gaussian

Elimination . . . . . . . . . . . .

An Error Analysis for Numerical Multiple Integration. I. . . . . 98

An Error Analysis for Numerical Multiple Integration. II..... 286

A Fast Fourier Transform Algorithm Using Base 8 Iterations. . 27.5

On Numerical Calculation of Transonic Flow Patterns....... 13

Powers of a Matrix of Special Type $(\mathrm{N}) \ldots \ldots \ldots \ldots \ldots \ldots 667$

More Amicable Numbers $(\mathrm{N}) \ldots \ldots \ldots \ldots \ldots \ldots \ldots \ldots 67$

See: BAkr, R. M. \& Brock, P... . . . . . . . . . . . . . . 38;

Bivariate Interpolation of Potential Functions. . . . . . . . . . 589

Substitution Formulas for Laplace Transformations (N) . . . . 209

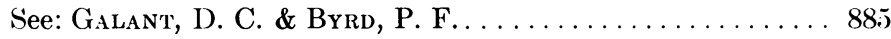

The Stability of Difference Approximations to a Self-Adjoint

Parabolic Equation, Under Derivative Boundary Conditions. 336

Polynomial System Satisfying a Special Functional Equation (N) 673

Explicit Inverses and Condition Numbers w. Certain Circulants

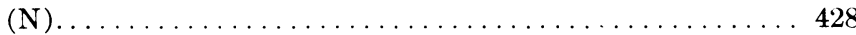

Error Estimates for Gauss Quadrature Formulas for Analytic

Functions . . . . . . . . . . . . . . . . . . 82

Asymptotic Error Estimates for the Gauss Quadrature Formula 91

Error Estimates for the Clenshaw-Curtis Quadrature........ 651

Error Bounds for the Gauss-Chebyshev Quadrature Formula of the Closed Type $(\mathrm{N}) \ldots \ldots \ldots \ldots \ldots \ldots \ldots \ldots . \ldots \ldots$

The Maxima of $P_{r}\left(n_{1}, n_{2}\right)(\mathrm{N}) \ldots \ldots \ldots \ldots \ldots \ldots \ldots \ldots$

On the Evaluation of Integrals Related to the Error Function. 137

Cho, Choong Yun

On the Triangular Decomposition of Cauchy Matrices...... 819

Chorin, Alexandre Jofl Numerical Solution of the Navier-Stokes Equations......... . 745

Churchrouse, R. F. See: Good, I. J. \& Churchrouse, R. F. . . . . . . . . . 857

Cody, W. J.

CoDY, W. J. \&

Chebyshev Approximations for the Fresnel Integrals (N).... 450

Rational Chebyshev Approximations for the Exponential

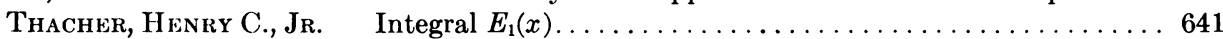

CoOpre, G. J.

DAHLQUist, G., SJÖBERG, B. \& Svensson, P.

Interpolation and Quadrature Methods for Ordinary Differential Equations............................ 69

Comparison of the Method of Averages with the Method of Least Squares............................. 833 
Author

DAwE, JAMES

DiCKFY, RTT

Eisen, Dennis

FARISS, R, R.

Fenchter, Christophrr A. Numerical Integration Over a Sphere.

FLeckNer, Oscar I.

Fosdick, LLOYd D.

Galant, D. C.

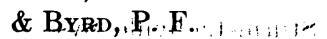

GaUtschi, WaLTer

Gizbert, R,E.

Glasser, M, L.

Goop, I. J. \&

- Churchounsw, R. F, F,

Gerdon, Payl

Gourlay, A. R. \& MORRIS, J. LL.

Gourlay, A. R. \& Morris, J. LL.

Gourlay, A. R. \& MORRIS, J. LI.

GRAY, H. L.

GraY, H. L. \& Atchison, T. A.

Gross, W! \&

: $\mathbf{V}_{\mathbf{A C C} A}, \mathbf{R}$.

Gupta, H.

HAGEMAN, L A \&

KELLOGG, R. B.

HANSEN, EILDON R.

HerRiot, J. G.

Hirsch, P. M.

Hunter, D. B.

$\mathrm{J}_{\mathrm{AIN}}, \mathrm{M} . \mathrm{K}$.

JAIN, M. K.

Jamet, Pifrre

Jamet, Pilrre

Johnson, Lee

JOHDAN, T. $\mathbf{k}$.

Julius, R. S.

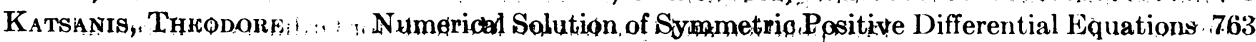

KEAST, P.

KELISKY, IR. P: \&

RIVLIN, T. J.

KELLOGG, R. B.

Title

Page

See: LAL, MUHÁ

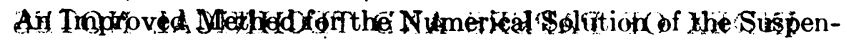

sion Bridge Deflection Equations............... 298

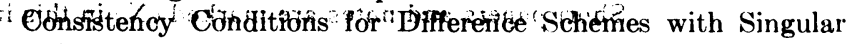
Coefficients........................... 347

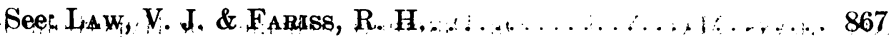

A Method for the Computation of the Fresnel Integrals and : : ? Related Functions .................................63

A Special Case of the Filon Quadrature Formula ........... 7 \$

High Accuracy Gamma Function Values for Some Rational,

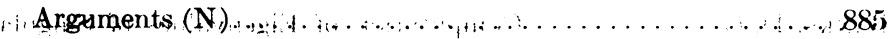

Construction of Gauss-Christoffel Quadrature Formulas........ 251

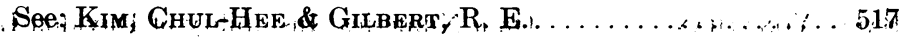

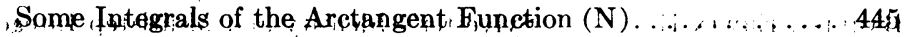

The Riemann Hypothesis and Psendorandom Features of the

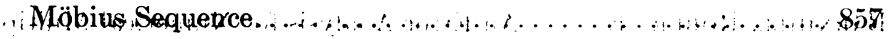

A Nate on a Maximum Principle for the DuFort-Franked I Piffer-

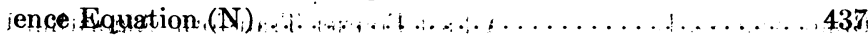

;Finite-Difference Methods for ANonhinear Hyperbolic Systems: . 28

Finite-Difference Methods for Nonlinear Hyperbolic Systems. I 49

A Multistep Formulation of the Optimized Lax-Wendroff Method for Nonlinear Hyperbolic Systems in Two Space :

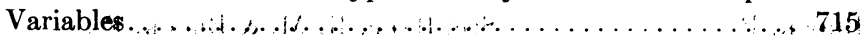

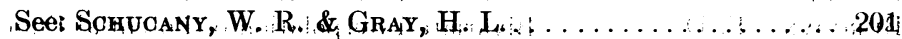

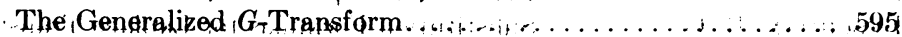

Distribution of the Figures 0 and 1 , in the Various Onders of $w^{\prime}$, )

Binary Representations, of $k$ th Powers of Integers (N)...... . 423

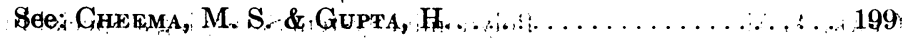

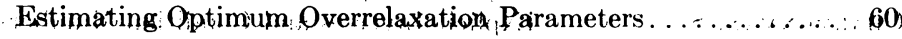

On Solving Systems of Equations Using Interval Arithmetic. 374;

See: Bergman, S., Herriot, J. G G \& Kurtz, T. G. . . . . . . . 13

Evaluation of , Orthogonal / Rolymomials and Relationship to : ;

Evaluating Multiple Integrals.............. . \% ..., $: 280$

The Evaluation of ia Class of Functions, Defined by an Integral, ,

$(\mathbf{N})$.

See: ChaWLA, M.. M. \& JAIN, M. K. $\mathbf{K}_{4}, \ldots \ldots \ldots \ldots \ldots \ldots .82$

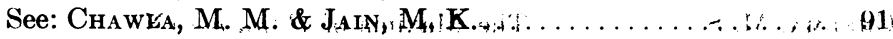

Finite Difference Methods for the Computation of thei l'Poissons . Kernel"'? of Elliptic Operators. ... $\ldots \ldots ! \ldots \ldots \ldots \ldots \ldots \ldots, 4, \ldots 7$,

Numerical Methods and Existence Theorems for Parabolic Differential, Equations, Whose Coefficients are Singular on the

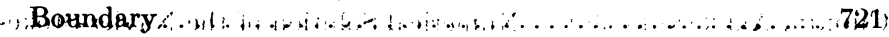

Unieity in Approximation of a Funetion and its Derivatives, (N) 873

Experiments on Error Growth Assogiated with Some Linear...

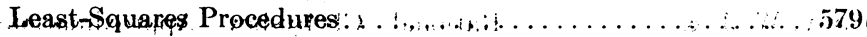

See: Charmonman, S. \& Jutius, R. $\mathrm{B} \ldots \ldots \ldots \ldots \ldots \ldots \ldots \ldots \ldots 428$

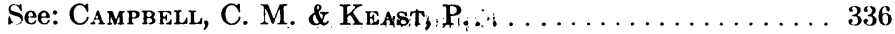

A Rational Approximation to the Lagarithm................ 128

See: Hagman, L. A. \& Kellogg, R. B. . . . . 
" Author

Kim, Chul-Hee \& Gillbert, R. E.

KLopfenstein, R. W. \& Millman, R. S.

Kreiss; Heinz-OtTo \& LUNDQVIsT; EINAR Kreiss, Heing OtTo

KunowskI, G. J.: Kortz, T. G. Lal, Mohan \& DAWE, JAMES

LiAw, V; J. \& FARISS, R. H.

Lice, Elvin J. LENiNe, NorMan LUKe, YUdell L. LUNDQVIST, EINAR.'! LUNNON, W. F: LETHER, H. A.

Lrach, Robert E. \& RICE, John R.

EYNESS, J. N. McKAY, JoHN Madey, Richard MARK, JohN MarkhaM, T. L.

Mrсhen, Fr.

Mrlzak, Z. A. MaLler, JoHN

Mulluman, R. S. Malnes, Harold Wiluis

Nitcheld, W. C. MorRIs, J. LK. Morris, J. LI. Morris, J. LL. Oldham, K. B. Osborn, DAví \& 1 MADEY, RICHARD Parlett, Beresford

Patterson, T. N. I. Pitterson, T. N. I. Price, Harvey S.

\section{Rabinowitz, Phimp}

Raichel, A.

Rice, JoHn R. \& Usow, KarL H.
Title

Page

Exaluation of Kernel Associated! with Laminar Flow Tubular : ., ;

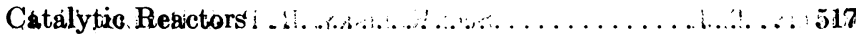

Numerical Stability of a One-Evaluation/Predietor-Correctior Algorithm for Numerical Solution of Ordinary Differential

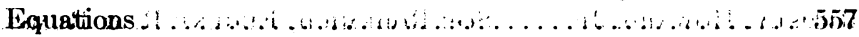

On Difference Apptoximations with Wrong Boundary Vialues a

Stability : Thebry for Differende Approximations of Mixedi Initial

Boundary. Value Problems $\mathbb{L} \ldots \ldots \ldots \ldots \ldots$

See: Banks; D: O. \& Kunowski, G.J.t . . . . . . . . . . 304

See: Bergman, S., Herriot, J. G. \& Kurtz, T. G. . . . . . . . 13

Solutions of the Diophantine Equation $x^{2}-D y^{4}=k(\mathrm{~N}) \ldots .679$

Or l the Relations Between Eigenvalues and Eigenvedtors for Matrices Resulting from Pre and Post Maltiplication by the

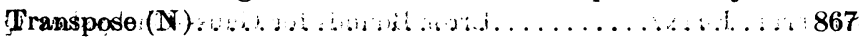

Amicabde aNumbers and the Bilinear Diophantine Equation : 181

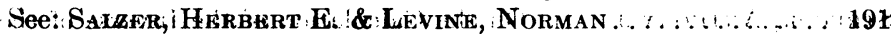

Approximations for Edliptic Integrals $\ldots \ldots \ldots \ldots \ldots \ldots \ldots \ldots \ldots$

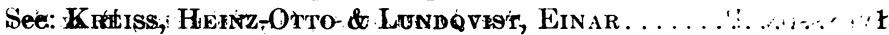

A Map Folding Broblem (N) $\ldots \ldots \ldots \ldots \ldots \ldots$

An Explicit Sixth-Order Runge-Kurtta Formulà (N)

Convergence :Rates of ADV Methods with Smooth Initid Error 311

Differentiation Formulas for Analytic Functions . . .

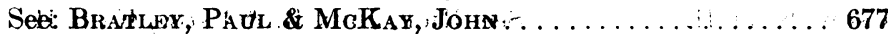

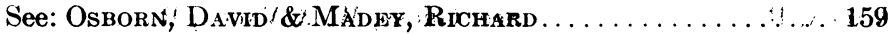

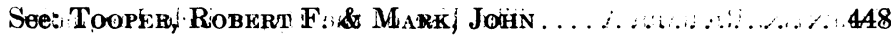

An Iterative Procedure for Computing the Maximal Root of a

Positive Matrix $(\mathbf{N}) \ldots \ldots \ldots \ldots \ldots \ldots \ldots \ldots$

Improvement in Recurrence Techniques for the Computation of Bessel Fundtions bf Integral Order $(\boldsymbol{N}) \ldots \ldots \ldots \ldots 2 . \ldots \ldots 2$

Numerical Evaluation of an Isoperimetric Constant . . . . . . . . 188

On the Resolvent of a Linear Operator Associated with a Well-

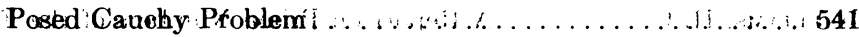

Seé: Klopfenstein, R. W: \& Miliman, R. S. . . . . . . $\ldots 557$

A Note Concetning the Properties of a Certain Class of Test

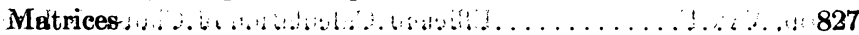

An Evaluation of Golomb's Constant. . . . . . . . . .

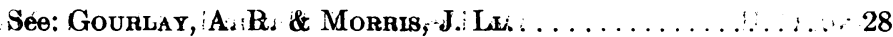

See: Gourlay, A. R. \& Morris, J J . . . . . . . . . . . . . 549

See: Gourlay, A. R. \& Morris, J: LH.. . . . . . . . . . . . . . 715

Approximations for the $x \exp x^{2}$ erfc $x$ Function (N) . . . . . . 454

The Fncomplete Beta Function and its Ratio to the cómplete

Beta Function ...... . . . . . . . . . . . . . 159

Global Canvergence of the Basic $Q R$ Algorithm on Hessenberg

Matrices ......................... 803

The Optimum Addition of Points to Quadrature Formulae.. . . 847

On Some Gauss and Lobatto Based Integration Formulae (N). 877

Monotone and Oscillation Matrices Applied to Finite Difference

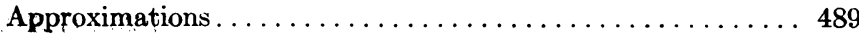

Error Bounds in Gaussian Integration of Functions of Low-

Order Continuity $(\mathrm{N}) \ldots \ldots \ldots \ldots \ldots \ldots \ldots \ldots \ldots \ldots \ldots \ldots$

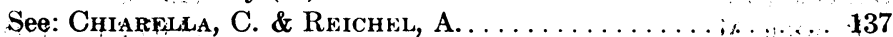

The Lawson Algorithm and Extensions............. 118 
Author

Rice, John R.

Rivlin, T. J.

Rodemich, Eughen R. \& Rumsey, Howard, Jr.

Rumsey, Howard, Jr.

Salzer, Herbert E.

\& Levine, Norman

SARMA, V. L. N.

Schucany, W. R. \& GraY, H. L.

SJöberg, B.

SPIRA, Robert

Spitznagel, Edward L., Jr. A Computer Study of the Orders of Finite Simple Groups (N). 669 \& SzYGENDA, STEPHEN A.

STHTTER, Franz

StetTer, Franz

Strecok, Anthony J.

STroud, A. H.

Svensson, P.

Swartz, Blitr K.

Szygenda, Sthphen A.

Thacher, Henry C., Jr.

Tooper, Robert F.

\& MARK, JohN

Usow, KarL H.

VACCA, R.

Valentin, Richard A.

VARAH, J. M.

VARAH, J. M.

Wilcox, Patter H.

Williams, H. C.

\& $Z_{\text {ARNKE, }}$ C. $R$.

WIMP, JET

WOOD, VAN E.

WrenCH, JoHN W., Jr.

YANG, C. H.

ZarNke, C. R.

Title

l'age

See: Lynch, Robert E. \& Rice, John R............... 311

See: Kelisky, R. P. \& Rivlin, T. J............... 128

Primitive Trinomials of High Degree $(\mathrm{N}) \ldots \ldots \ldots \ldots \ldots \ldots . \ldots 63$

See: Rodemich, Eugene R. \& Rumsey, Howard, Jr....... . 863

Proof that Every Integer $\leqq 452,479,659$ is a Sum of Five

Numbers of the Form $Q_{x} \equiv\left(x^{3}+5 x\right) / 6, x \geqq 0(\mathrm{~N}) \ldots \ldots .191$

Eberlein Measure and Mechanical Quadrature Formulae. I:

Basic Theory.......................... 607

A New Approximation Related to the Error Function (N).... 201

See: Dahlquis', G., SJöberg, B. \& Svensson, P........ . . 83:3

Zeros of Sections of the Zeta Function. II............. 163 A.

Error Bounds for Gauss-Chebyshev Quadrature (N) . . . . . 6.57

On a Generalization of the Midpoint Rule (N) .........661

On the Calculation of the Inverse of the Error Function...... 144

Extensions of Symmetric Integration Formulas.......... 271

See: Dahlquist, G., SJöberg, B. \& Svensson, P......... 833

Explicit $O\left(h^{2}\right)$ Bounds on the Eigenvalues of the Half-L.... 40

See: Spitznagel, Edward L., Jr. \& Szygenda, Strephen A... 669

See: Cody, W. J. \& Thacher, Henry C., Jr........... 641

Simplified Calculation of $\operatorname{Ei}(x)$ for Positive Arguments, and a

Short Table of $\operatorname{Shi}(x)(\mathrm{N}) \ldots \ldots \ldots \ldots \ldots \ldots \ldots \ldots \ldots \ldots \ldots$

See: Rice, John R. \& Usow, KarL H............... 118

See: Gross, W. \& VACCA, R................... 423

The Use of the Hypercircle Inequality in Deriving a Class of

Numerical Approximation Rules for Analytic Functions..... 110

The Calculation of the Eigenvectors of a General Complex

Matrix by Inverse Iteration. . . . . . . . . . .

Rigorous Machine Bounds for the Eigensystem of a General

Complex Matrix............................. 793

The Zeros of $P_{\nu}{ }^{1}(\cos \theta)$ and $(\partial / \partial \theta) P_{\mu}{ }^{1}(\cos \theta)(\mathrm{N}) \ldots \ldots \ldots \ldots 20$.

A Report on Prime Numbers of the Forms $M=(6 a+1) 2^{2 m-1}$

-1 and $M^{\prime}=(6 a-1) 2^{2 m}-1(\mathrm{~N}) \ldots \ldots \ldots \ldots \ldots \ldots 420$

Recursion Formulae for Hypergeometric Functions. . . . . . 363

Efficient Calculation of Clausen's Integral $(\mathrm{N}) \ldots \ldots \ldots \ldots . \ldots 83$

Concerning Two Series for the Gamma Function........6 617

On Designs of Maximal $(+1,-1)$-Matrices of Order $n \equiv 2$

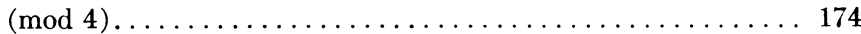

See: Williams, H. C. \& Zarnke, C. R............... 420

\section{INDEX OF REVIEWS BY AUTHOR OF WORK REVIEWED}

\begin{tabular}{|c|c|c|}
\hline Author & Review Number & Classification \\
\hline Ascari, Aldo & 84 & $(7.20 .2)$ \\
\hline Avdeyeva, L. I. & 38 & $\begin{array}{l}\text { See: Zukhovitskiy, S. I. } \\
\text { \& Avdeyeva, L. I. }\end{array}$ \\
\hline Babister, A. W. & 11 & $\begin{array}{l}(4.10 .1) \\
(5.05 .1),(5.10 .1) \\
(7.25),(7.30),(7.35),(7.40),(7.45), \\
\quad(7.50),(7.55),(7.65),(7.90),(7.105)\end{array}$ \\
\hline $\begin{array}{l}\text { Babuska, I., } \\
\text { Prager, M. } \\
\text { \& VitaseK, E. }\end{array}$ & 9 & $\begin{array}{l}(2.55) \\
(4.05 .2 .1),(4.05 .3 .1),(4.10 .1 .1) \\
\quad(4.10 .2 .1),(4.10 .3 .1) \\
(5.05 .3 .1),(5.10 .2 .1)\end{array}$ \\
\hline
\end{tabular}

Page

898

457

223

Prager, M.

$(2.55)$,

$(4.10 .2 .1),(4.10 .3 .1)$,

(.5.05.3.1), (5.10.2.1) 
Author

Bailey, Leslie F.

Bates, Frank

$\&$ Douglas, Mary L.

Bauer, Charles R.,

Peluso, Anthony P.

\& Worley, William S., Jr.

Beckett, Royce

\& HuRT, James

Beiler, Albert H.

Beliakov, V. M.,

KravTSOVA, R. I., \& RapPaPORT, M. G.

Bellman, R. E.,

KaLABA, R. E.,

\& LOCKETT, J.

Berger, Bruce S., \& Danson, Robert

Birkhoff, GarretT

Bork, Alfred M.

Brand, Louis

Burckhardt, Johann Jakob

Caputo, Anthony J.

Caslin, James C.

Chretien, M., \& Deser, S., Editors

Collatz, Lothar

Collatz, Lothar

\& Wetterling, Wolfg.ing

Concus, Paul

Cruon, R., Editor

DANson, Robert

Davis, Philip J. \& Rabinowitz, Philip

Dawe, James

Dawe, James

Dekanosidze, E. N.

Deser, S.
Review Number

Classification

Page

35

(2.05.3)

(12.05.1)

$(12.05 .1)$

455

237

26

37

(2.05.3), (2.10), (2.20), (2.35),

(3.05), (3.10), (3.15), (3.30),

(4.05), (4.10), (4.15),

(5.10.2)

74

(9.00)

(7.60), (7.95)

690

231

6

(2.10.1),

(3.25),

(6.15),

(7.90)

40

(2.10)

458

76

32

See: MacLane, Saunders

\& Birkhoff, Garrett

(12.05.1),

(13.05)

83

(4.00),

(6.05)

33

(13.05)

See: Thatcher, Charles M. \& Caputo, Anthony J.

18

See: Fettis, Henry E. \& Caslin, James C.

60

(13.05)

215

693

242

898

242

240

230

470

(2.05), (2.35),

(3.05),

(4.10), (4.15),

(5.10.2.1), (5.15.2.1),

(6.15), (6.35)

4

(2.05.2),

215

(3.25),

(5.10)

(2.20)

(8.10),

(13.35)

See: Berger, Bruce S.

\& Danson, RoBert

(2.10.1), (2.10.2), (2.10.3), (2.35) 459

See: LaL, MohaN

235

\& DAWE, JAMES

See: Lal, Mohan

\& Dawe, JaMes

(2.10.1),

(7.50)

See: Chretien, M.

462

689

458

692

460

60

\& Deser, s., Editors 


\begin{tabular}{|c|c|}
\hline \multirow{2}{*}{$\begin{array}{l}\text { Author } \\
\text { Worn, William S., } \\
\& \text { GREEnBERG, HeRBert J. }\end{array}$} & \\
\hline & $\begin{array}{l}\therefore \ldots 442 \\
\therefore \because 41\end{array}$ \\
\hline & 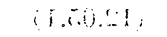 \\
\hline $\begin{array}{l}\text { OUGLAS, MARY L. } \\
\text { and }\end{array}$ & $\therefore \quad{ }^{25}$ \\
\hline $\begin{array}{l}\text { RAPER, NoRMaN } \\
\text { \& SMITH, HaRRY }\end{array}$ & $\begin{array}{l}11,60) 62 \\
1 i t, 60.1\end{array}$ \\
\hline Uris, Charles S. & $\begin{array}{c}(6.0 ! .79 \\
(6,0) 6 !\end{array}$ \\
\hline $\begin{array}{l}\text { Die, Leshie C., } \\
\text { HeRMan, RoBert } \\
\text { \& Rothery, RichaRd, Editor }\end{array}$ & $80,-159$ \\
\hline NGLANDER, HLRman S. & 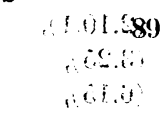 \\
\hline GLANDER, W & $\begin{array}{l}689 \\
111.6\end{array}$ \\
\hline
\end{tabular}

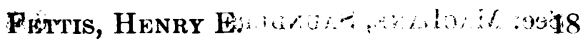

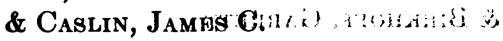

Fleckner, Oscar L. H.

Foster, J. M. M.

GKMKRELIDZe, R. V., Editor

Glasmacher, Werner

Y \& Sommer, Dietmar and:

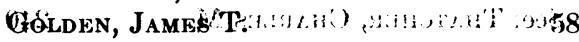

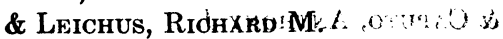

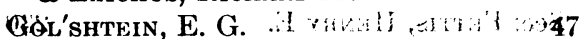
$\therefore$ and $x+1,0 \%$

Grienderg, Herbert J. $\mathbf{J}$. 42

Grienspan, Donald, Editor

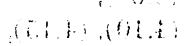

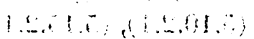

Guter, R. S., and (a) a 1

$\therefore$ Kudryavtsef, L. D., \& Levitan, B. M.

HALE, JACK K. , in

Hartmanis, J. 10.9 (is, 6$)$ (1). 49 va.: a.c.

\& STEARNS, R. E.

Herman, Robert and and

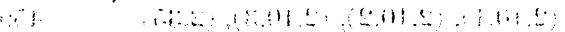

Hintze, Guenther Hollingdale, S. H., Editớ $: \cdots \cdots 4 y_{i} 34$ Hứt, JaMES JoHN, F.

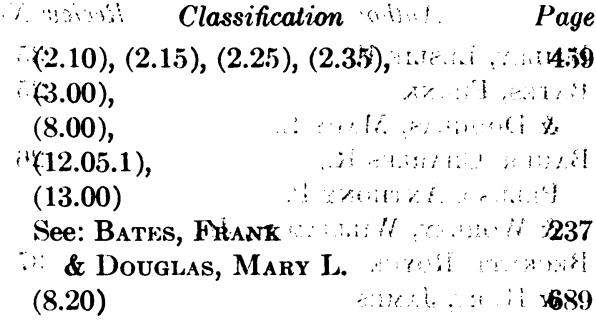

See: Moursund, David G. $\quad 894$ F \& Duris, Charles S. ? (13.00)

a n

isee: SAXon, James A., ㄱ. ENGLANDER, HrRMAN $\$$ \& Englander, William R, a i See: SAXon, James A., $\quad 902$

(1) Englander, Herman 8. \& ENGLANDER, WILATAM R. $(7.60),(7.95) \quad$,

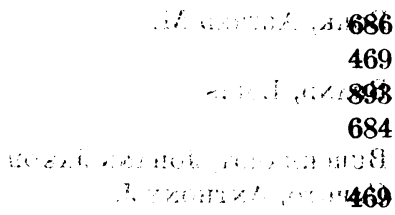

$(-12.05 .1)$ ) $\div \ldots: 4,42$

See: Yudin, D. B. \& Gol'shtein, E. (i.

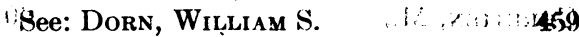
\& Greenberg, Hardiher. 5 .

(3.25), (4.05.3.1), (4.15.1.1), (4.15.3.1),

(5.10.2.1), (5.20.2.1),

(13.15), (13.35)

(2.05), (2.05.2), (2.05.3) 212

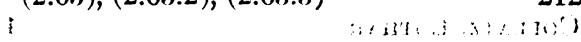

(4.00), 463

(5.00),

$(13.15),(13.35) \quad$..it $\ldots . .1 \%)$

(12.00),

(13.35) : : : : \% : (1)

See: Edie, Leslie C., $\quad 470$

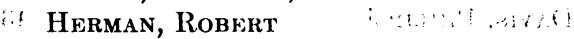

\& Rothery, Richakìd, Editors

(12.05.1), (12.05.2) … 241

(13.15), (13.35), (13.40) 243

See: Beckett, Royce

\& HURT, J AMES

(2.05.1), (2.05.3), (2.20), (2.35), 683

(3.10.1), (3.15.1), (3.20.1),

(4.05.3.1),

(5.05.2.1), (5.05.4.1), (5.10.2.1), (5.20.3.1) 


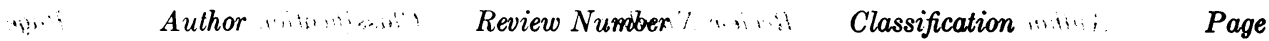

JoNES, M. F.

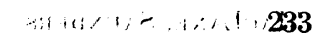

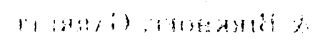

Jones, M. F.

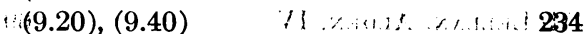

Falaba, R. E.? :

See: Bellman, R. E., . $6111,1 /$, , , , 215

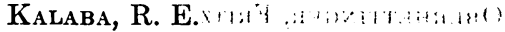

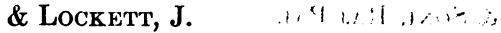

Karmazina, L. N. : . 54

!:i:

Kaufmann, A. . i. . $/ !$. . .

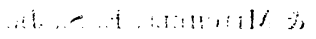

sivis.

Fere, Dorothy

i.:.

Khamis, S. H.

: i 155

ג.:

16

Klerer, Melvin

$\therefore ;$

$\therefore$ \& Korn, Granino A Editorsili,

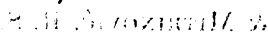

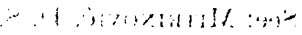

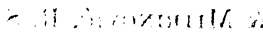

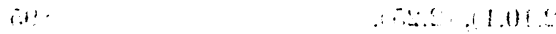

Korn, Granino A.

a.:.:

KravTsova, R. I.

$\therefore 19$

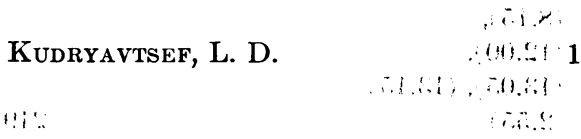

IHAL, M. $\quad \therefore \quad$ :

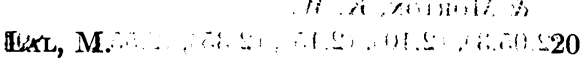

LAL, M. .

LaL, Mohan

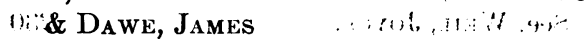

LaL, Mohan \& Dawe, James $\because 1$ :

Livi, M.

: ‘i). 86 \& LunNon, W. F. 68

LAL, M.

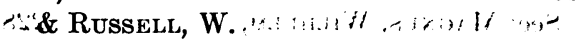

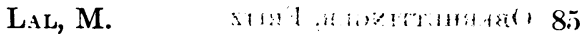

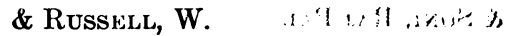

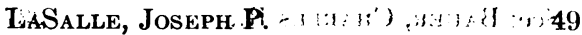

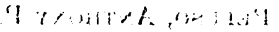

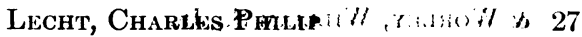

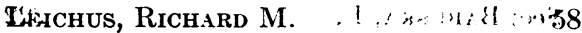

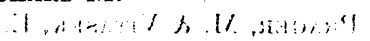

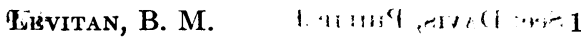

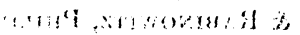

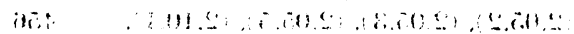

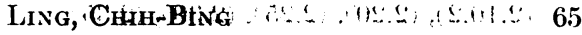

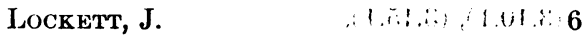

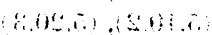

LunNon, W. F.
(See: $\overparen{Z}$ Hurina, M. I. :

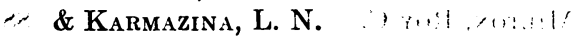

$\therefore(3.25)$, (10.35),

i. $(13.35)$

178.05)

$\therefore \therefore, 1,1,1683$

$(7.20)$,

(8.50)

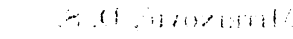

(2.05), (2.10), (2.15), (2.20), (2.55), i\%. 893

(. $(3.00)$,

(4.00),

(75.00),

(8.00),

(12.05.1), (12.05.2)

(13.15), (13.35), (13.40)

See: Klerer, Melvin 893

\& Korn, Granino A., Editors

See: BelIAKov, V. M., Kravtsova, R. I.

\& RAPPAPORT, M. G.

See: Guter, R. S.,

Kudryavtsef, L. D.

\& Levitan, B. M. \& 4 , .

(7.05),

il $, 1, \cdots, 226$

(9.40)

(79.05), (9.40)

(7.05)

1) (iit: (1, , : iris 2332

:!

(9.00)

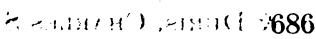

$23 \pi$

$-1,1,1,1, \% ?+21 \%$

692

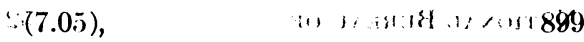

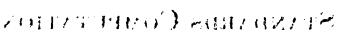

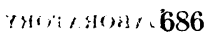

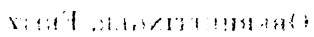

899

See: HaLE, JACK K. "† \& LaSalle, Joseph P., Editors

(12.05.1)

238

See: Golden, James T. $\quad 1 ! \ldots:, 469$ \& Leichus, Richard M.

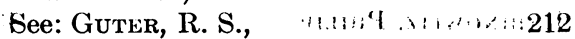
Kudryavtsef, L. D.

\% \& Levitan, B. M. immir. . A :

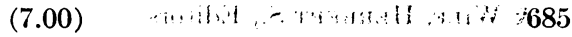

See: Bellman, R. E.,

KaLABA, R. E.

\& LockETT, J.

See: LAL, M. 


\begin{tabular}{|c|c|c|c|}
\hline Author & Review Number & Classification & Page \\
\hline $\begin{array}{l}\text { MacLane, SAUNdERS } \\
\text { \& BirkHOFF, GaRRETT }\end{array}$ & 76 & $(10.00)$ & 693 \\
\hline McLlellan, Alden, IV & 69 & $(7.00)$ & 687 \\
\hline $\begin{array}{l}\text { Magnus, Wilhelm, } \\
\text { Oberhettinger, Fritz } \\
\text { \& Soni, RaJ PaL }\end{array}$ & 15 & $\begin{array}{l}\text { (7.15), (7.20), (7.25.1), (7.30), } \\
\quad(7.40),(7.45),(7.50),(7.55),(7.60) \\
\quad(7.90),(7.105)\end{array}$ & 228 \\
\hline Michaud, Françoish & 50 & $(5.15)$ & 465 \\
\hline Mitton, Roy C. & 88 & $(8.35)$ & 901 \\
\hline Mitchell, E. S., JR. & 53 & $\begin{array}{l}\text { See: Wyndrum, R. W., Jr. } \\
\text { \& Mitchell, E. S., JR. }\end{array}$ & 467 \\
\hline MItrRinović, D. S. & 46 & $(2.50)$ & 462 \\
\hline $\begin{array}{l}\text { Mírinović, D. S. } \\
\quad \& \text { Mitrinović, R. S. }\end{array}$ & 70 & $(7.05)$ & 688 \\
\hline $\begin{array}{l}\text { Mitrinović, D. S. } \\
\quad \text { \& Mitrinović, R. S. }\end{array}$ & 71 & $(7.05)$ & 688 \\
\hline Mitrinović, R. S. & 70 & $\begin{array}{l}\text { See: Mitrinovic, D. S. } \\
\text { \& Mitrinović, R. S. }\end{array}$ & 688 \\
\hline Mitrinović, R. S. & 71 & $\begin{array}{l}\text { See: Mitrinović, D.S. } \\
\text { \& Mitrinović, R. S. }\end{array}$ & 688 \\
\hline Mond, Bertram, Editor & 81 & $\begin{array}{l}(2.10 .1),(2.25), \\
(3.25), \\
(4.05), \\
(5.05),(5.10),(5.15),(5.20), \\
(6.35), \\
(7.20 .2),(7.75), \\
(8.15), \\
(12.00), \\
(13.05),(13.15)\end{array}$ & 895 \\
\hline Moore, Ramon E. & 8 & $(2.55)$ & 219 \\
\hline Monton, K. W. & 51 & $\begin{array}{l}\text { See: Richtmyer, R. I). } \\
\text { \& Morton, K. W. }\end{array}$ & 465 \\
\hline $\begin{array}{l}\text { Moursund, David G. } \\
\text { \& Duris, Charles S. }\end{array}$ & 79 & $\begin{array}{l}(2.05 .3),(2.10),(2.15),(2.35),(2.55), \\
(3.00), \\
(4.05 .2 .1),(4.05 .3 .1)\end{array}$ & 894 \\
\hline Murty, Tadepalli $\mathrm{S}$. & 17 & $\begin{array}{l}\text { See: Weil, Joyce, } \\
\text { Murty, TadePalli s. } \\
\text { \& Rao, Desiraju B. }\end{array}$ & 230 \\
\hline $\begin{array}{l}\text { National Burfau of } \\
\text { Standards Computation } \\
\text { Laboratory }\end{array}$ & 52 & $(7.65)$ & 466 \\
\hline Oberhettinger, Fritz & 15 & $\begin{array}{l}\text { See: Magnus, Wilheli, } \\
\text { OberhetTINGER, Fritz } \\
\text { \& Soni, RAJ PAL }\end{array}$ & 228 \\
\hline Peluso, Anthony P. & 26 & $\begin{array}{l}\text { See: Bauer, Charles R., } \\
\text { Peluso, Anthony P. } \\
\text { \& Worley, William S., Jr. }\end{array}$ & 237 \\
\hline Prager, M. & 9 & $\begin{array}{l}\text { See: Babuska, I., } \\
\text { Prager, M. \& Vitasek, E. }\end{array}$ & 222 \\
\hline Rabinowitz, Philip & 43 & $\begin{array}{l}\text { See: Davis, Philip J. } \\
\text { \& Rabinowitz, PhiLiP }\end{array}$ & 459 \\
\hline $\begin{array}{l}\text { Raiston, Anthony } \\
\text { \& Wilf, Herbert S., Editors }\end{array}$ & 36 & $\begin{array}{l}(2.05 .2),(2.05 .3),(2.05 .5),(2.10 .1), \\
\quad(2.10 .2),(2.20),(2.25),(2.30),(2.35), \\
(3.10 .1),(3.15 .1), \\
(5.10 .2),(5.20 .3), \\
(7.30), \\
(8.05), \\
(12.05 .1)\end{array}$ & 456 \\
\hline
\end{tabular}


Author

Rao, Desiraju B.

RAPPAPORT, M. G.

Reed, Georgia B.

RICHTMYER, R. D. \& Morton, K. W.

Roosen-Runge, Peter H.

RoThery, RICHARD 87 59

Russell, W.

Russell, W.

SAXon, James A., Englander, Herman S. \& Englander, William R.

Shipley, HerberT

Shipley, Herbert

Simonnard, Michel

SMITH, HARRY

SмIтн, R. E.

SNYDer, M. A.

Sommer, Dietmar

Soni, RaJ Pal

STAKGOLD, Ivar

STEARNS, R. E.

Thatcher, Charles M. \& Caputo, Anthony J.

TOMPA, H.

Urabe, Minoru

Vitasek, E.

Vorobyev, Yu. V.

WALL, Francis J.

17

19

68

8.5

89

72

63

15

10

56
Review Number

Classification

Page

See: Wril, JoYce, Murty, Tadepalli S.

\& Rao, Desiraju B.

See: BelTAKov, V. M.,

KravTsova, R. I.

\& RAPpaport, M. G.

See: Weeg, Gerard P.

\& Reed, Georgia B.

(5.05.2.1), (5.05.3.1), (5.20.2.1), (5.20.3.1),

(6.35)

(7.00),

(10.00)

See: Edie, Leslie C.,

Herman, Robert

\& Rothery, RichaRd, Editors

See: Lal, M. \& Russell, W.

See: Lal, M. \& Russell, W.

(12.05.1)

230

231

214

465

900

470

686

899

902

(7.10),

227

(13.15.1)

(7.10),

227

(13.15.1)

(3.25),

(9.00),

(10.35),

(13.15), (13.30)

See: Drapkr, Norman

\& SMITH, HARRY

(12.05.1)

(2.05.2), (2.05.5),

(6.05),

(7.30), (7.55)

See: Glasmacher, Werner

\& Sommer, Dietmar

See: Magnus, Wilhelm,

Oberhettinger, Fritz

\& Soni, RAJ PaL

(4.10.1),

(6.15)

See: Hartmanis, J.

\& Stearns, R. E.

463

(12.05.1)

689

239

894

684

228

223

235

240

(2.10.1) $\quad 459$

(4.00) 684

See: Babuska, I., Prager, M. 222

\& VitaseK, E.

(2.35),

218

(4.10),

(5.10.2), (5.10.3),

(6.15), (6.35),

(13.15)

(8.15) 


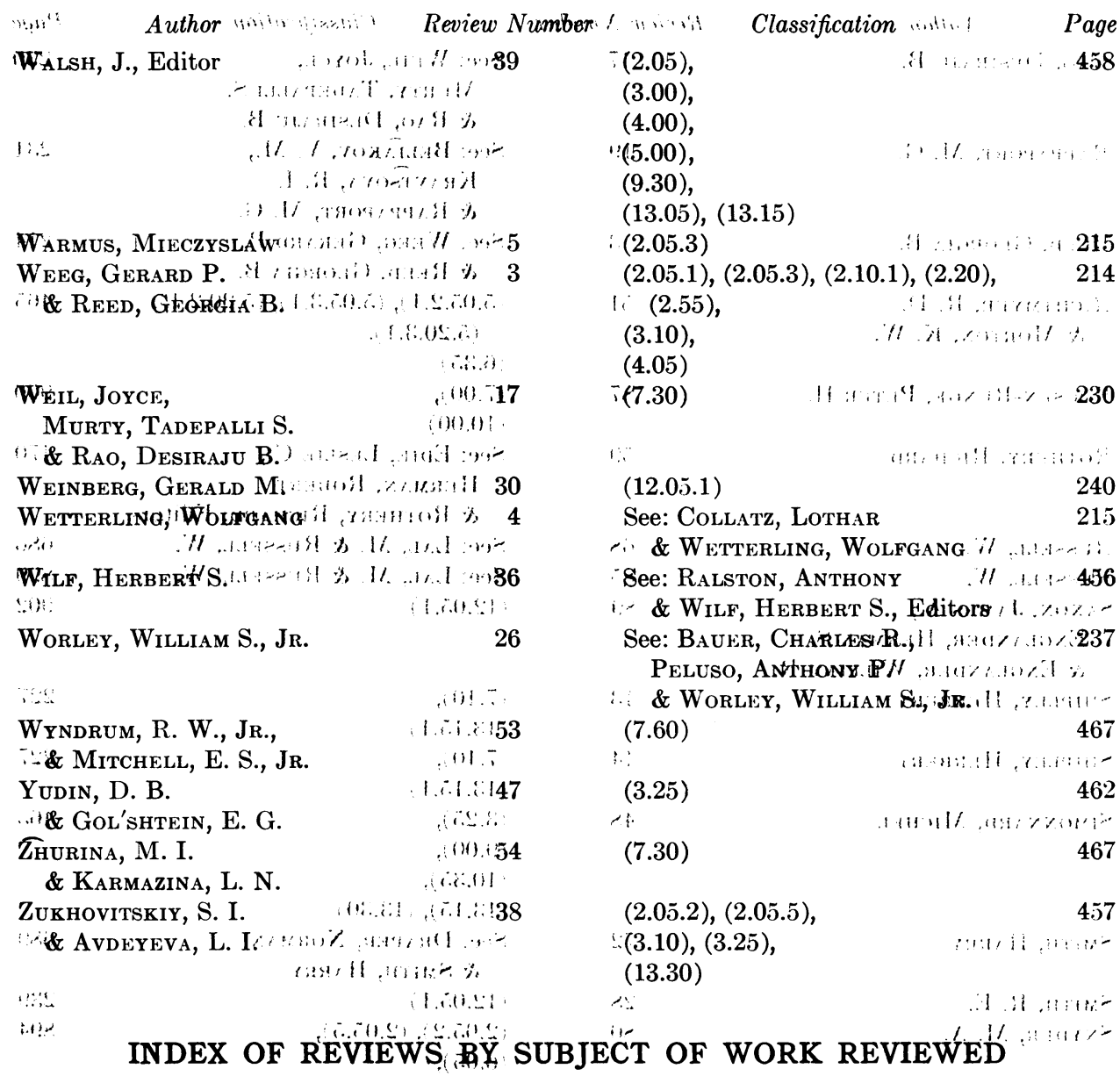

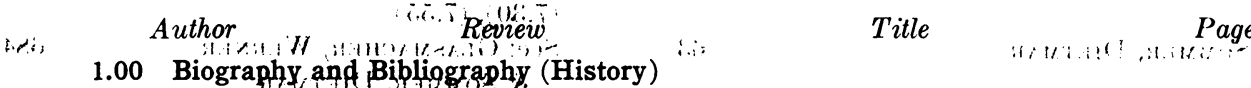

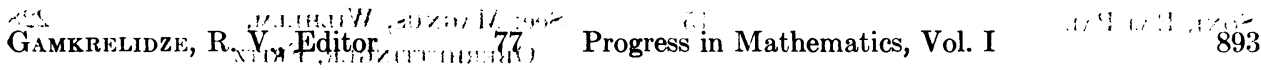

$$
\therefore \quad 2.00 \text { Selected Topics in Numerical Analysis }
$$

a.s. 2.05 Approximation Theory.

Collatz, Lothar $\quad$ if il , - i:, ir $\%$ 2, Functional Analysis and Numerical

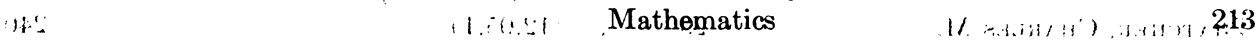

Klerer, Melvin $\quad 78$ Digital Computer User's Hapdbopk 1 , i i,,$\$ 93$

\& Korn, Granino A., Editors 11.01.:

WaLSH, J., Editor 391, Numeriqad Analysis: An Introduction

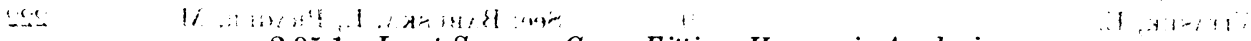
2.0.). i Least Squanes, Curve Fitting, Harmonic Analysis

Jown, F. $\quad$,61..... Lectureš on Advanced Numerical Analysis : $1:$, 683

Weeg, Gerard P. Ba Introduction to Numerical Analysis 214

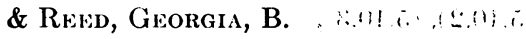

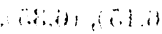

2.05.2 Chebyshev.(Best) Approximation

Cobllatz, Lothar

\& Whtterling, Wolfgang

i. Optimierungsaufgaben 
GUTeR, R. S.,

1 . Elements of the Theory of Functions, .:1, : if , 212

KUDRYAVTSEF, I. D:

\& Levitan, B. M.

RALSTON, ANTHON i:? in:

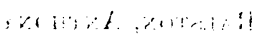

(36) ,Mathematical Methods for Digital Computers

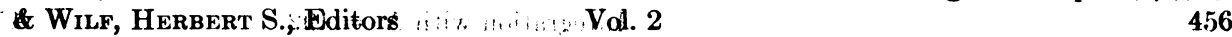

SNyder, M. A. 80 Chebyshev Methods in Numerical Approxima-

ZuKHovitskiY, S. I. tion

\subsubsection{Interpolation, Extrapolation}

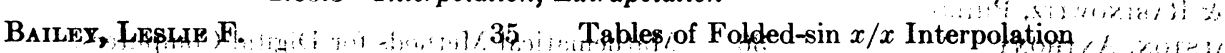

BECKETT, RoYCE

\& HURT, JAMES

Gứrer, R. S.,

KudRyavtsef, L. D.

\& Levitan, B. M.

JoHN, F.

Moursunp, DaNid, G.

- \& Duris, Charles S.

RaLSTON, ANTHONX \& Wilf, Herbert S., Editors

Warmus, Miegrastaw

Wheg, Gerard P.

\& Reed, Georgia B.

61 Lecturesion Advancod Numerical Analysis 683

79 Elementary Theory and Application of

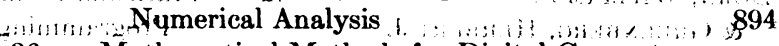

$36_{1}$ Mathematicat-Methods for Digital Computers

1. Interpolations In

3 Introduction to Numerical Analysis

\section{Coefficients}

$37 \quad$ Numerical Calculations and Algorithms

of the Theory of Functions

1. Elemepts of the Theory of Functions

$\therefore$ ? An: and :

214

\subsubsection{Rational Approximatión} Ralston, AnThony
\& WILF, Herbert S., Editors

SNYDER, M. A,

80. Chebyshev Methods in Numerical Approxima-

ZUKHOVITSKIY, S. I. \& AvDFYey, 38 Linear and Convex Programming

\subsection{Numerical Integration}

Bécketr, Rorce \& HURT, JAMES

Berger, Bruce S. \& Danson, RoBerT

40 Tables of Zeros and Weights for Gauss-Laguerre Quadrature

Jorn, WiLliam $\mathrm{S}$. ratkénatics and Computing: with Fortran 458 \& Greenberg, Herbert J. ， Programming 459

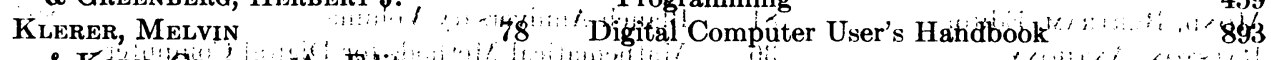
\& KónN, GRANino Á., Editors

Moursund, David G. \& Duris, Charles $\mathrm{S}$.

79 Elementary Theory and Application of $1 ! \ldots$ Numerical Analysis

\subsubsection{One-Dimensional i :}

Beillman, R. E., KaLABA, R. E. \& LOCKETT, J.

1) Avis, Philri J." \& Rabinowitz, Philip

DEKANOSIDZE; $\mathbf{N}$

6 Numerical Inversion of the Láplace Transform 215

43) Numerical Integration

'44 Tablitsy kornei i vesovykh mnozhitelèi obobshchennykh polinomov Lagerra (Tables of the zeros and weight factors of the generalized Laguerre polynomials) 
Author

Mond, Bertram, Editor

RALSTON, ANTHONY

\& Wilf, Herbert S., Editors

Tомга, $\mathrm{H}$.

Weeg, Gerard P.

\& Reed, Georgia $B$.
Review Title

81 Blanch Anniversary Volume

Page

36 Mathematical Methods for Digital Computers, Vol. 2

41 Abscissae and Weight Factors for Gaussian Integration with $N=192$

3 Introduction to Numerical Analysis

\subsubsection{Multi-Dimensional}

Davis, Philip J.

\& Rabinowitz, Philip

RALSTON, ANTHONY

\& Wilf, Herbert S., Editors
43 Numerical Integration

36 Mathematical Methods for Digital Computers, Vol. 2

Davis, Philip J. \& Rabinowitz, Philip

459 2.10.3 Monte Carlo

\subsection{Numerical Differentiation}

Dorn, William S. \& Greenberg, Herbert J.

KLerer, Melvin 42 Mathematics and Computing: with Fortran Programming

78 Digital Computer User's Handbook

\& Korn, Granino A., Editors

Moursund, David G. \& Duris, Charles S.

$79 \quad$ Elementary Theory and Application of Numerical Analysis

894

\subsection{Roots of Equations}

Beckett, Royce \& Hurt, James

Concus, PaUl

JoHn, F.

KleRer, Melvin \& Korn, Granino A., Editors

RALSTON, ANTHONY \& Wilf, Herbert S., Editors

Weeg, Gerard P. \& Reed, Georgia B.

\subsection{Evaluation of Series}

Dorn, William S. \& Greenberg, Herbert J.

Mond, Bertram, Editor

Ralston, ANTHONY \& Wilf, Herbert S., Editors
$37 \quad$ Numerical Calculations and Algorithms

45 Table of the Solutions of $a \tan (\pi x)=$ $-b \tan (a \pi x)$

78 Digital Computer User's Handbook

36 Mathematical Methods for Digital Computers, Vol. 2

3 Introduction to Numerical Analysis

\subsection{Continued Fractions}

Ralston, ANTHONy

\& Wilf, Herbert S., Editors

Mathematical Methods for Digital Computers, Vol. 2

2.35 Iteration Methods, Acceleration Techniques

BecketT, Royce \& HURT, JAMES

Collatz, Lothar

Davis, Philip J. \& Rabinowitz, Philip
$37 \quad$ Numerical Calculations and Algorithms

2 Functional Analysis and Numerical Mathematics 213

43 Numerical Integration 
Author

DORN, WiLliam S. \& Greenberg, Herbert J.

JoHn, F.

Moursund, David G.

Ralston, Anthony \& Wilf, Herbert S., Editors

Vorobyev, Yu.V.

\subsection{Inequalities}

Mitrinović, D. S. \& Duris, Charles S.
Review Title

42 Mathematics and Computing: with Fortran Programming

61 Lectures on Advanced Numerical Analysis

79 Elementary Theory and Application of Numerical Analysis

36 Mathematical Methods for Digital Computers, Vol. 2

7 Method of Moments in Applied Mathematics

$46 \quad$ Nejednakosti (Inequalities)
Page

459

683

\subsection{Stability of Computation, Significance Arithmetic}

Babuska, I., Prager, M.

9 Numerical Processes in Differential Equations \& Vitasek, E.

Klerer, Melvin \& Korn, Granino A., Editors

78 Digital Computer User's Handbook

Moore, Ramon E.

Moursund, David G. \& Duris, Charles S.

Weeg, Gerard P. \& Reed, Georgia B.

8 Interval Analysis

79 Elementary Theory and Application of Numerical Analysis

3 Introduction to Numerical Analysis

894

214

\subsection{Linear Algebra}

Dorn, William S. \& Greenberg, Herbert J.

Klerer, Melvin \& Korn, Granino A., Editors

Moursund, David G. $\&$ Duris, Charles S.

WALsh, J., Editor

Mathematics and Computing: with Fortran Programming

78 Digital Computer User's Handbook

79 Elementary Theory and Application of Numerical Analysis

39 Numerical Analysis: An Introduction

\subsection{Matrices}

Beckett, Royce
\& Hurt, James
Collatz, Lothar

37 Numerical Calculations and Algorithms

2 Functional Analysis and Numerical Mathematics

\subsection{Linear Equations}

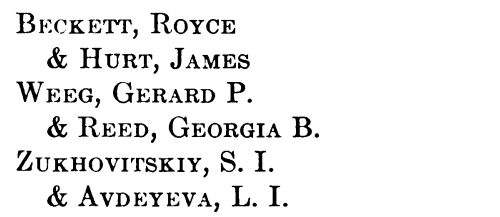

\subsubsection{Error Analysis}

John, F.

Ralston, Anthony

\& Wilf, Herbert S., Editors
61 Lectures on Advanced Numerical Analysis

36 Mathematical Methods for Digital Computers, Vol. 2

\subsection{Eigenvalues and Eigenvectors}

Becketr, Royce \& Hurt, James
$37 \quad$ Numerical Calculations and Algorithms

3.15.1 Error Analysis

JoHn, F.

61 Lectures on Advanced Numerical Analysis 
1.0 Author Review $\quad$ Title rin: Page

Ralston, ANTróny: 36 Mathehatical Methods for Digital Computerts,

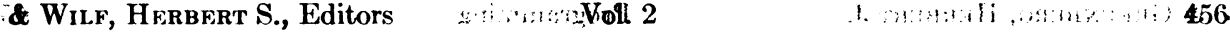

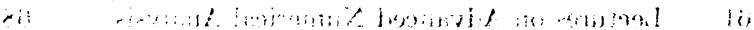
3.20 Matria Inverswonand Pseudo-Ihvierses

:!e rimlant inomanth

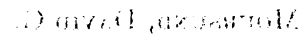

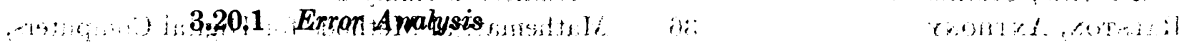

Join, F.

61 Ledtures on Advanced Nitumerical inglystis 1 il 683

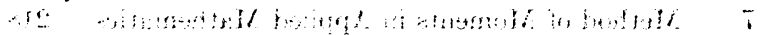

3.25 Linear and Nonlinear Programming, Theory of Games

Bellman, R. E., Kalaba, R. E. 6 Numerical Inversion of the liaplace Transform 215

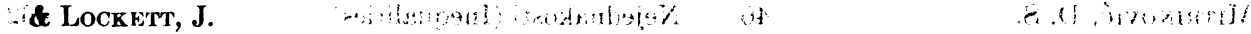

Collatz, Lothar 4 Optimierungsaufgaben

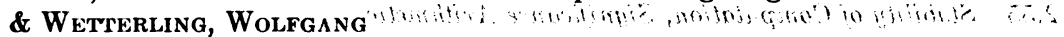

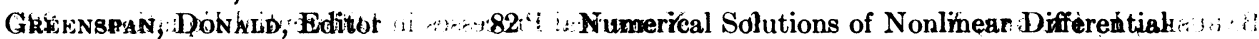
Equations

$\therefore$.

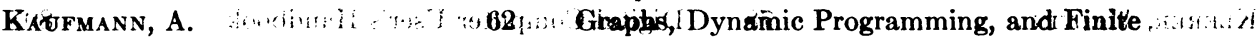
Games

Mdnd, Ber'ram, Editor $\quad$ 81: Blameh Anniversary Volume $\quad$. Simonnard, Mogkz

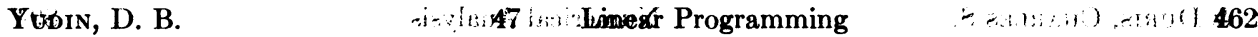

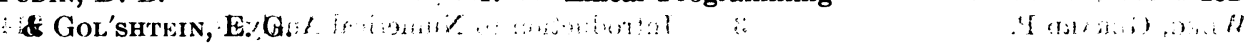
ZukhovitskiY, S. I.

$38 \quad$ Linear and Convex Programming \& Avdeyeva, L. I.

adgoik rsonil on.દ.

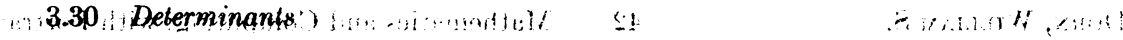

Bééentr, Royce

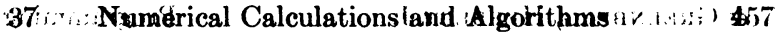

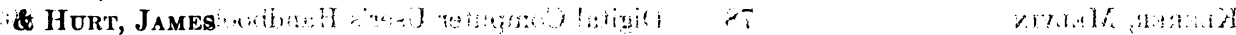

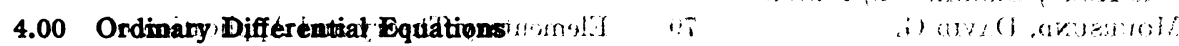

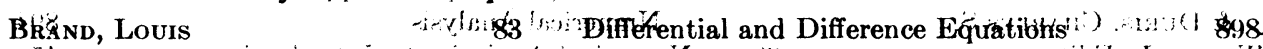
Gritsmacher, Witivek \& SOMMER, Dietmar

HALE, JACK K.

\& LASALLE, JoSEPH P., Editors 49 , Differential Equations and Dynamical Systems 463 KLeRer, Melvin.
\& Korn, Granino A., Edítors

STAKGold, IVAR

10 Boundary Value Problems of Mathematical

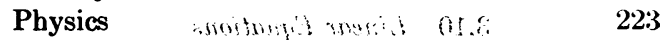

URABe, Minord WALSH, J., Editor $\quad 39 \quad$ Numerical Analysis: An Introduction 4.05 Initial Value Problems minlal a if (nombi), and

BECKeTr, Royce \& HURT, JAMES Mond, Bertram, Editor

81 Blanch Anniversary Volume 895 Weeg, Gerard P.

3 Introduction to Nuñerical Analysis 214

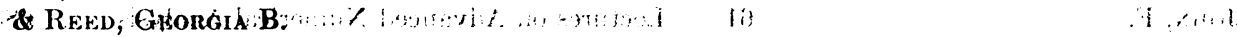

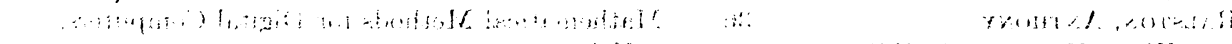
4.05.2 One-Step Methods

4.05.2.1 Error Analysis: 1 hurn

Babuska, I., Prager, M. Numerical Processes in Differential Equations 9222 \& VitaseK, E.

Moursund, David G.

Elementary Theory and Application of Numerical Analysis 
Author $\quad$ Review

4.05.3 Multistep Methods

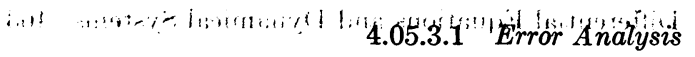

insule Title wits

Page

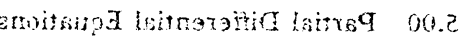

it

A

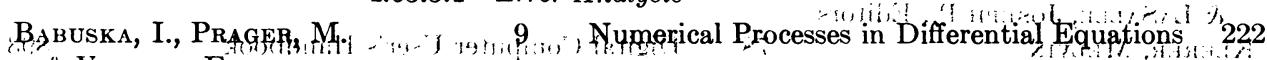
\& VitaseK, E.

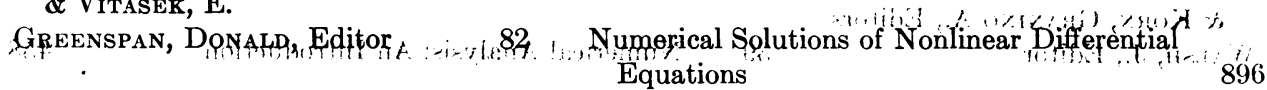

JонN, F. $\quad 61 \quad$ Lectures on Advanced Numerical Analysis 683

Moursund, David G.
\& Duris, Charles S.

4.10 Linear Boundary Value and Eigenvalue Problems

BeCEetr, Rgyce Numerical Calculations and Algorithms 457 \& HURT, JAMES

Collatz, Lothar

2 Fúnctional Analysis and Numerical

Vorobyev, YU. V. Mathematips min a

7 Method of Moments in Applied Mathematics

4.10.1 Analytic Methods

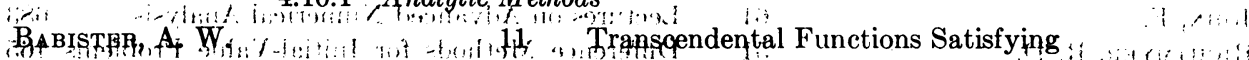

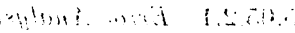 Nonhomogeneous Linear Differential Equations
Stakgold, Ivar
10w: Howndary Kalue Prablems of Mathematical Physics

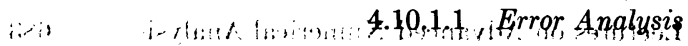
9 Numerical Processes in Differential Equations 222
Babuska, I., Prager, M. $\quad 9$ Numerical Processes in Differential Equations 222 \& ViTASEK, E. andu? 4 an' i.:
i.:
4.10.2 Initial Value (Trial and Error or Shooting), Methods

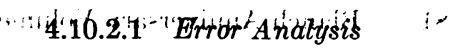 (1)

Babuska, I., Prager, M. \& VITASEK, E.

$9 \quad$ Numerical Processes in Differential Equations 222

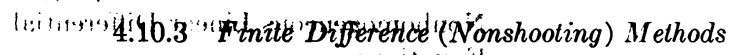

$\because \cdots$

\subsubsection{Error Analysis}

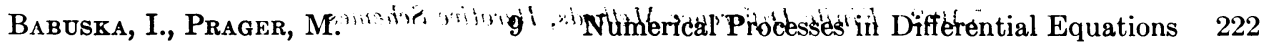

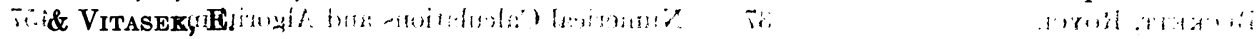

4.15, Aloplinean Bopmdary, Value and Eigenqalue Problems

$$
\begin{aligned}
& \text { - In: . . 1: } 11 \%
\end{aligned}
$$

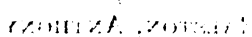

B.PCETT, ROYCE

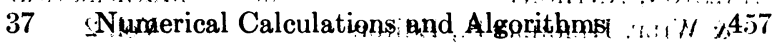

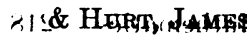

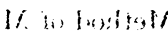

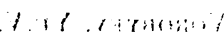

Collatz, Lothar

2 Functional Analysis and Numerical

Mathematios 1 : $: 1 ! 1: \therefore$

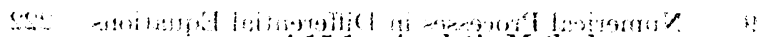

4.15.1 Analytic Methods

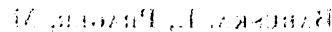

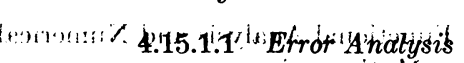

$\therefore, d+1,1 / 2$,

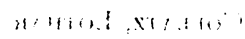

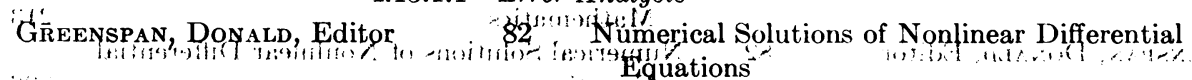

inie [ti) Equations

896

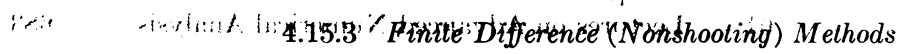

$1 \ldots, \cdot$,

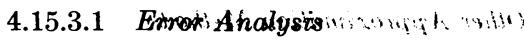

Greenspan, Donald, Editor

82 Numerical Solutions, of Nonlinear Differential

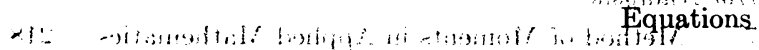




$$
\text { Author Review Title Page }
$$

\subsection{Partial Differential Equations}

HALE, JACK K.

\& LaSalle, Joseph P., Editors

KLERER, MELVIN

\& Korn, Granino A., Editors

WALSH, J., Editor
49 Differential Equations and Dynamical Systems 463

78 Digital Computer User's Handbook

893

$39 \quad$ Numerical Analysis: An Introduction

\subsection{Initial Value Problems}

Mond, Bertram, Editor

81 Blanch Anniversary Volume

895

5.05.1 Analytic Methods

Babister, A. W.

11 Transcendental Functions Satisfying

Nonhomogeneous Linear Differential

Equations

\subsubsection{Explicit Difference Methods}

\subsubsection{Error Analysis}

JoHN, F.

61 Lectures on Advanced Numerical Analysis 683

RiCHTMYER, R. I).

51 Difference Methods for Initial-Value Problems 465

\& MorTon, K. W.

\subsubsection{Other Approximation Methods}

\subsubsection{Error Analysis}

JoHN, F.

61 Lectures on Advanced Numerical Analysis

\subsection{Boundary Value Problems}

Collatz, Lothar

\& WeTterling, WolfGang

Mond, Bertram, Editor
4 Optimierungsaufgaben

81 Blanch Anniversary Volume
895

\subsubsection{Analytic Methods}

Banister, A. W.

11 Transcendental Functions Satisfying Nonhomogeneous Linear Differential Equations

5.10.2 Finite Difference Methods, Iterative Schemes

BeCketT, Royce \& HURT, JAMES

RaLSTON, ANTHONY

\& Wilf, Herbert S., Editors

Vorobyev, Yu.V.

$37 \quad$ Numerical Calculations and Algorithms

36 Mathematical Methods for Digital Computers, Vol. 2

$7 \quad$ Method of Moments in Applied Mathematics

\subsubsection{Error Analysis}

Babuska, I., Prager, M. \& VitaseK, E.

Collatz, Lothar

Greenspan, Donald, Editor

JoHn, F.
$9 \quad$ Numerical Processes in Differential Equations

2 Functional Analysis and Numerical Mathematics

82 Numerical Solutions of Nonlinear Differential Equations

61 Lectures on Advanced Numerical Analysis
222

457

\subsubsection{Other Approximation Methods}

\subsubsection{Error Analysis}

Vorobyev, Yu.V.

7 Method of Moments in Applied Mathematics 
Author Review Title Page

5.15 Eigenvalue Problems

Mond, Bertram, Editor

Michaud, Françoise
81 Blanch Anniversary Volume

50 "Sur le calcul numérique des valeurs propres de l'équation de Hill"

895

465

\subsubsection{Finite Difference Methods, Iterative Schemes}

\subsubsection{Error Analysis}

Collatz, Lothar

2 Functional Analysis and Numerical Mathematics

5.20 Mixed Initial and Boundary Value Problems

Mond, Bertram, Editor

81 Blanch Anniversary Volume

895

\subsubsection{Explicit Difference Methods}

\subsubsection{Error Analysis}

Gremspan, Donald, Editor

$82 \quad$ Numerical Solutions of Nonlinear Differential Equations

Richtмyer, R. D.

51 Difference Methods for Initial-Value Problems \& Morton, K. W.

\subsubsection{Implicit Difference Methods, Iterative Schemes}

$\begin{array}{ccc}\text { Ralston, Anthony } & 36 & \text { Mathematical Methods for Digital Computers, } \\ \text { \& Wilf, Herber' S., Editors } & & \text { Vol. } 2\end{array}$

\subsubsection{Error Analysis}

JoHN, F.

61 Lectures on Advanced Numerical Analysis

RICHTMYER, R. D.

51 Difference Methods for Initial-Value Problems

\& Morton, K. W.

\subsection{Other Functional Equations}

Collatz, Lothar

2 Functional Analysis and Numerical

Mathematics

\subsection{Difference Equations}

Brand, Louis

SNYDER, M. A.
83

80
Differential and Difference Equations

Chebyshev Methods in Numerical Approximation

\subsection{Integral Equations}

Bellman, R. E.,
\& LocketT, J.

Collatz, Lothar

STAKGOLd, IvaR

Vorobyev, YU.V.
6 Numerical Inversion of the Laplace Transform

2 Functional Analysis and Numerical Mathematics

10 Boundary Value Problems of Mathematical Physics

$7 \quad$ Method of Moments in Applied Mathematics

6.35 Abstract Operator Equations

Collattz, Lothar

Mond, Bertram, Editor

RICHTMYER, R. D. \& Morton, K. W.

VововуеV, YU.V.
2 Functional Analysis and Numerical Mathematics

81 Blanch Anniversary Volume 895

51 Difference Methods for Initial-Value Problems 465

$7 \quad$ Method of Moments in Applied Mathematics 


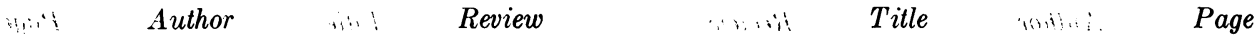

7.00 Special Functions

Eleckner, Oscar L. $\quad 686$

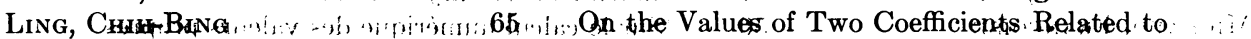
rial:

McLellan, Alden, IV

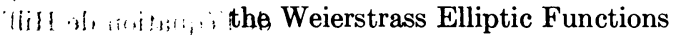

685

69 Tables of the Riemann Zeta Function and

A Table of Bell Polynomials: $Y_{1}$ to $Y_{10}$

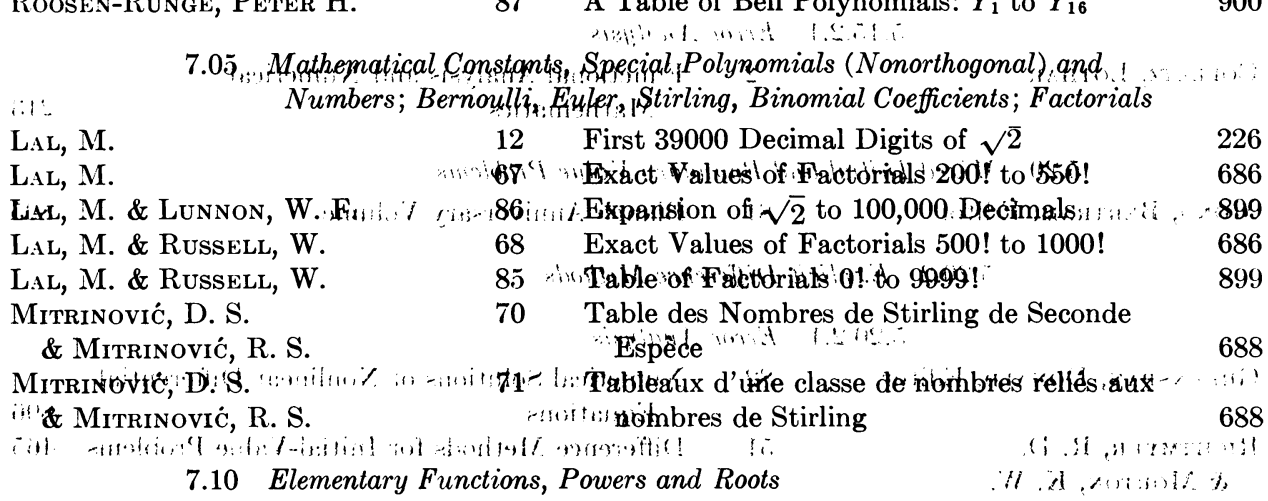

Shipley, Herbert $\quad 13$ Standard Tables for Circular Curves 227

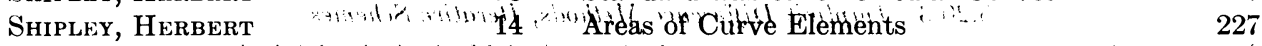

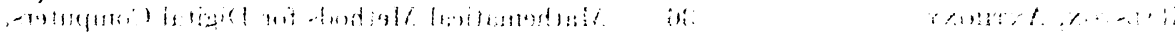

ii $\quad 7.15$ Gamma Functions, Psi Functions, Zeta Functions and Related Finctionsi औ,

Magnus, Wilhelm,

OberhetTinger, Fritz

$15 \quad$ Formulas and Theorems for the Special

Fundions of Mathematical Physics

are SonI, RAy: PAL

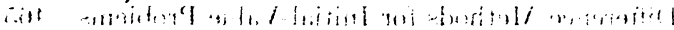

7.20 Incomplete Gamma Functions

(4) 11 , in 1 (r)

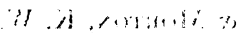

Khamis, S. H. $\quad 16$ Tables of the Incomplete Gamma Function

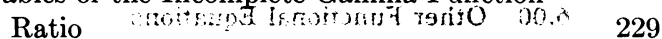

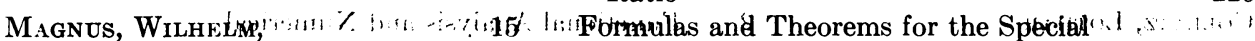

i. Oberhettinger, Fritz $\quad 21 \%$

\& Soni, RAJ PAL

Mond, Bertram, Editor

81 Blanch Anniversary Volume $\quad 895$

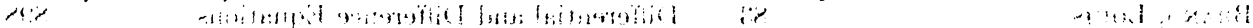

1,7i:20.2 $/$ Errot, Fitometions, Fresnel Integrals and Related Functionts $1 / \ldots$

Alscari, Aldo

:! 84 :

Function

898

Mond, Bertram, Editor

81 Blanch Anniversary Volume

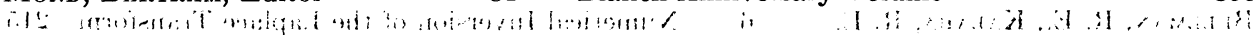

7.25 Confluent Hypergeometric Functions

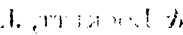

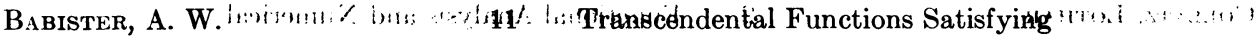
i.1.

$\because$ ilisamilonhomogeneous Linear Differential

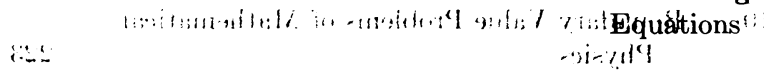

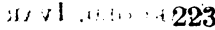

16 :

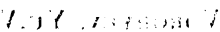

Magnus, Wilhelm,

Oberhettinger, Fritz

15 Formulas and Theorems for the Special

Functions of Mathematical Physids

\& SonI, RAJ PAZ

i1 $11: \div ;$

$1, ;$

i:?

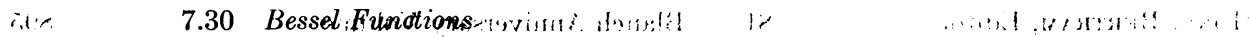

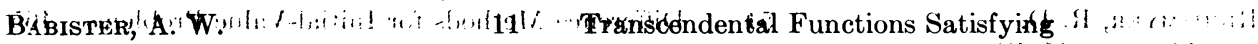

Nonhomogeneous Linear Diffeftental

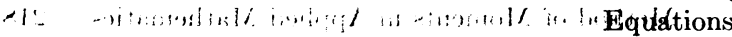


Author Review Title Page

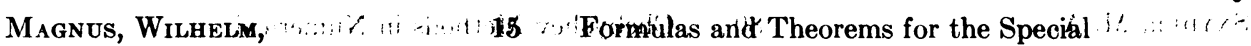

Oberhettinger, Fritz $\quad 228$

\& SonI, RAJ PAL

Ralston, Anthont

\& Wilf, Herbert S., Editors Vol. $2 \quad 456$

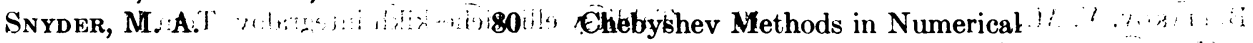

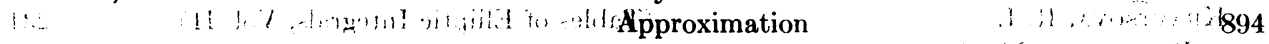

Wril, JoYce, $\quad 17$ Zeros of $J_{n}(\lambda) Y_{n}(\eta \lambda)-J_{n}(\eta \lambda) Y_{n}(\lambda)$ and ili.

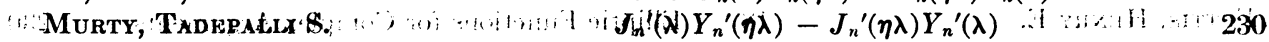
\& RaO, Desiraju B.

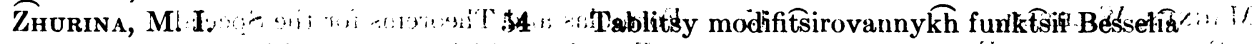

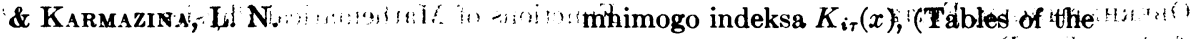
modified Bessel function of imagiharyl , irot $t$,

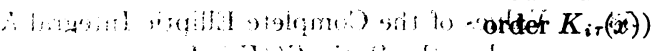

7.35 Lommel Functions, Struve Functions, Anger-Weber Functions and

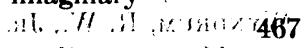

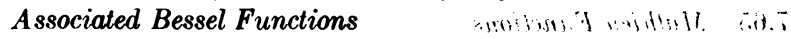

Babister, A. W w (tiw) Equations

7.40 Tegendre Functions

Babister, A. W.

Transcendental Functions Satisfying

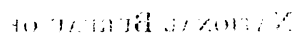
Nonhomogeneous Linear Differential

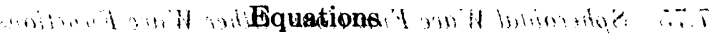

Magnus, Wilhelm, ...1, $\%$ 15, Formulas and Theorems for the Special $\begin{array}{lll}\text { Oberhetringer, Fritz } & \text { Functions of Mathematical Physies } & 228\end{array}$ \& SoNI, RAJ PAL

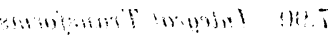

\subsection{Gássian Hypergeométric Functions}

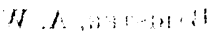

BABISTER, A. W:

$\therefore \quad$ B. Nonhomogeneous finear Differential

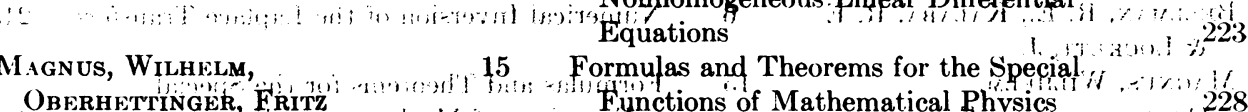

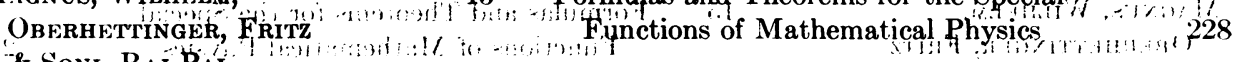
\& SoNI, RAJ PAL

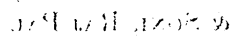

\subsection{Orthogonal Polynomials and Functions}

Babister, A. W. Transcendental Functions Satisfying

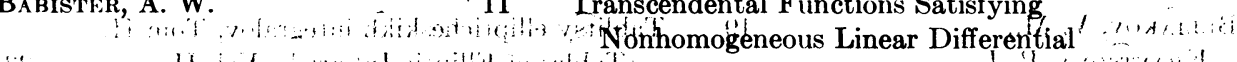

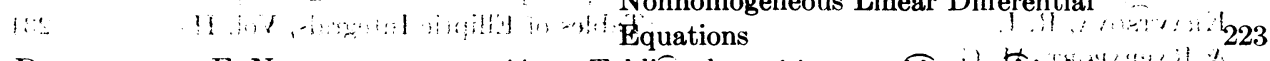

Drkanosidze, E. N.

44 Tablitsy kornei i vesovykh mónhitelè

;

obobshénnykh polinomov Lagerra (tablest of the zeros and weight factors of the generalized Laguerre polynomials).

Magnus, Wilhelm, 15 Formulas and Theorems for the Special Oberhetringter Frtrz \& SoNI, RAJ PA'

\subsection{Expansions in, Beries:of Onthagonal Polynomials, Bessel Functions!! :- ; i...}

$\because \quad$ A W Other Functions an an

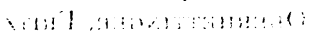

Babister, A. W.

11 Transcendental Functions Satisfying Nonhomogeneous Linear Differential

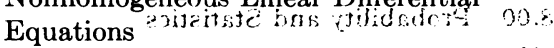
460

BA

MagnUS, WhídecM

15 Formulas and Theorems for the Spectial

Oberhettinger, Fritz

Functions of Mathetnatical Physics 


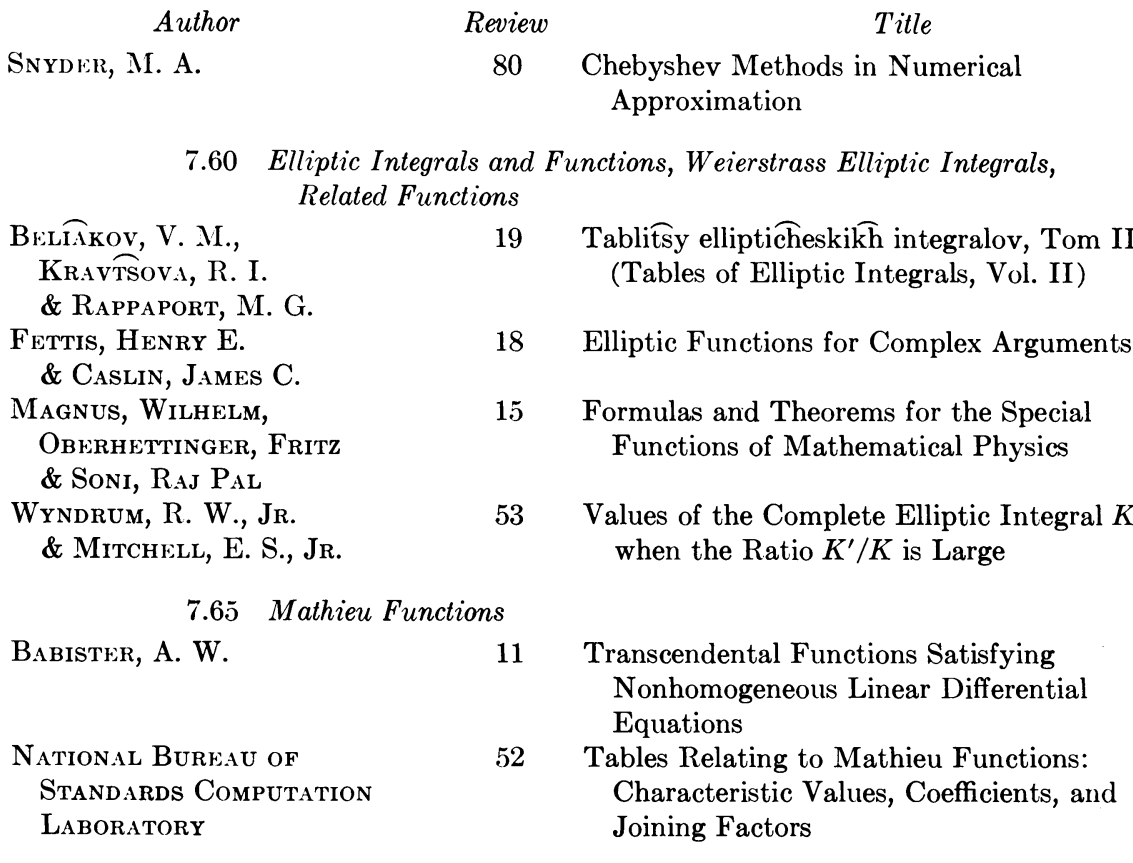

\subsection{Spheroidal Wave Functions. Other Wave Functions}

MOND, BERTRaM, Editor

81 Blanch Anniversary Volume

Page

\subsection{Integral Transforms}

BABISTER, A. W.

11 Transcendental Functions Satisfying Nonhomogeneous Linear Differential Equations

Bellman, R. E., KALABA, R. E. 6 Numerical Inversion of the Laplace Transform \& LockeTr, J.

Magnus, Wilhelm, Oberhettinger, Fritz

15 Formulas and Theorems for the Special Functions of Mathematical Physics

\& Soni, RAJ PAL

\subsection{Numerical Tables of Integrals and Transforms}

BELIAKOv, V. M., KRAVTSOVA, R. I. \& RAPPAPORT, M. G.

Fettis, Henry E. \& Caslin, James C.

19 Tablitsy ellipticheskikh integralov, Tom II, (Tables of Elliptic Integrals, Vol. II)

\subsection{Handbooks of Mathematical Tables and Formulae}

Babister, A. W.

Magnus, Wilhelm, Oberhettinger, Fritz \& Soni, RAJ PAL
11 Transcendental Functions Satisfying Nonhomogeneous Linear Differential Equations

15 Formulas and Theorems for the Special Functions of Mathematical Physics

\subsection{Probability and Statistics}

Dorn, William S. \& Greenberg, Herbert J. Programming 
\& Korn, Granino A., Editors

\subsection{Handbooks of Tables and Formulae}

KH.Mis, S. H.

16 Tables of the Incomplete Gamma Function Ratio

\subsection{Number Theory}

Bailer, Albert H.

74 Consecutive Hypotenuses of Pythagorean Triangles

Lal, Mohan

75. Solutions of the Diophantine Equation

\& Dawe, James $x^{2}-D y^{4}=k$

Simonnard, Michel

$48 \quad$ Linear Programming

\subsection{Mersenne, Fermat, Perfect, and Related Numbers}

L.AL, M.

20 Decimal Expansion of Mersenne Primes

9.10 Number Theoretic Functions and Tables

Jones, M. F.

21 Isoperimetric Right-Triangles

\subsection{Primes and their Distribution}

JoNes, M. F.

22 22900D Approximation to the Square Roots of the Primes less than 100

9.30 Forms and Diophantine Equations

LAL, MOHAN

\& DaWe, JaMES

WALSH, J., Editor
23

Tables of Solutions of the Diophantine

Equation $x^{2}+y^{2}+z^{2}=k^{2}$

39 Numerical Analysis: An Introduction

\subsection{Normal Numbers and Distribution of Digits}

JoNes, M. F.

LAL, M.

LAL, M.

LaL, M. \& LunNon, W. F.
22900D Approximation to the Square Roots of the Primes less than 100

86 Expansion of $\sqrt{2}$ to 100,000 Decimals 


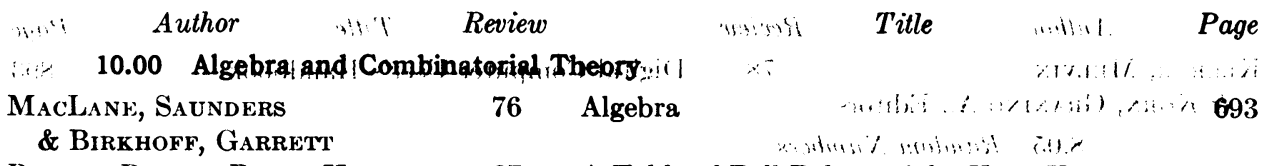

Roposen-Runge, Peter H.

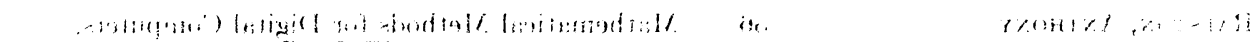

10.35 Graph Theory - $1.1 \%$ -

Kaufmann, A. 62 Graphs, Dynamic Programming, and Finite Gámes

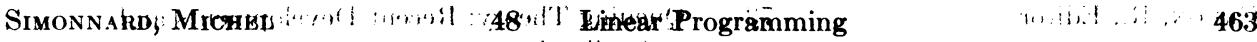

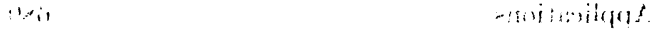

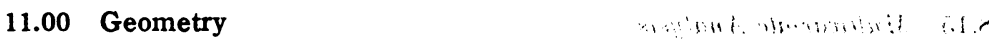

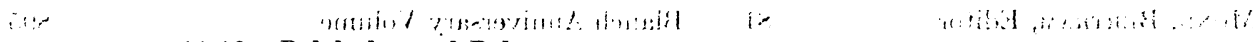

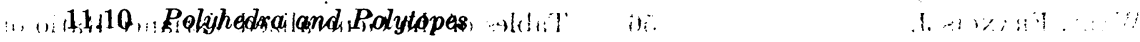

Joves, M. F. $\quad 21$, $\quad$ Isóperimetric Right-Triangles

233

12.00 Computers and Other Aids to Computation

HARTmanis, J.
\& Stearns, R. E.

Mond, Bertram, Editor $\quad 81 \quad$ Blanch Anniversary Volume

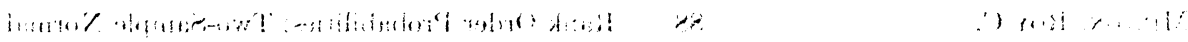

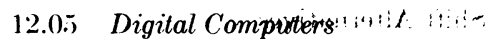

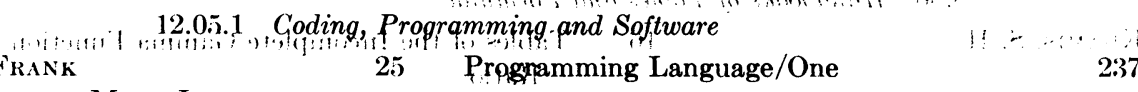

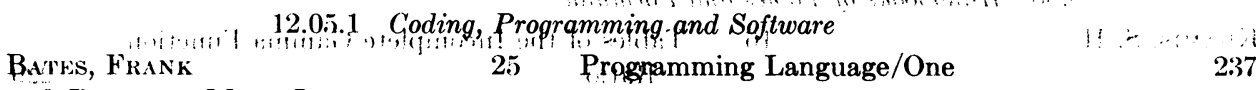

\& Douglas, Mary I.
Bork, Alfked M.

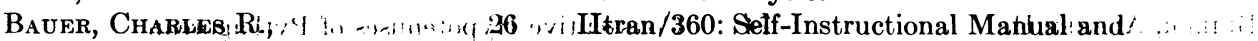

1: Afluso, Anthony P.

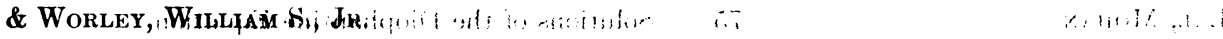

Dorn, William S. $\quad 142$ :

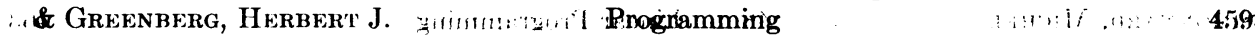

Foster, J. M. $\quad 57 \quad$ List Processing 469

Golden, James T. $\quad 469$

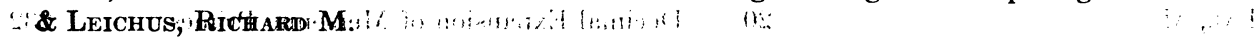

Hintze, Gojnther $\quad 31 \quad$ Fundamentals of Digital Machine Computing 241

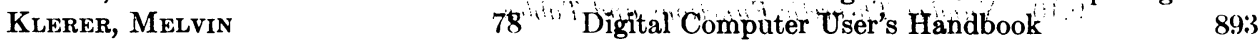

Korn, Granino A:, Dditors

Lecht, Charles Philip 27 The Programmers ALGOL: A Complete

Reference 238

Ralston, An'thör

H Wilf, Herbert S., Editots -

Saxon, James A., $\quad 89 \quad$ System 360 Programming $\quad 902$

Englander, Hraman S.

\& ENGLANDER, WHULHAM $\mathrm{R}$.

Sirth, R. E.
Tht 28 The Bases of Fortran

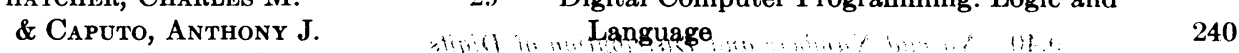

Weinberg, Gerald M.

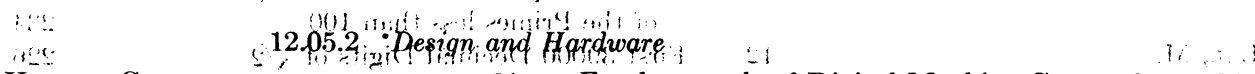

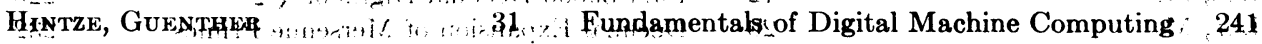

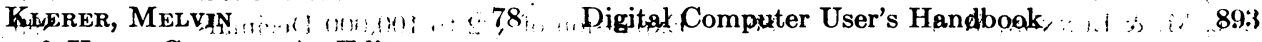
\& Korn, Granino A., Editors 
Author

\subsection{Applications}

İonn, Wrlliam $\mathrm{S}$. \& GREENBERG, 42 , Mathematics, and Computing: with Fortran Hrogramming J. Pis Epie, Leslie C.,

'Herman, RoberT

\& Rothery, RICHARD, Editors

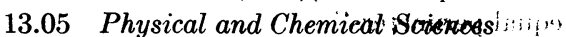

Börk, Alfred M.

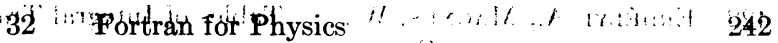

BURCKHARDT, JohaNn JaKoB 33 Die Bewegungsgruppert dèr Kristallographie

\& Deser, S., Editors

Mond, Bertram, Editor 81 Blanch Anniversary Volume

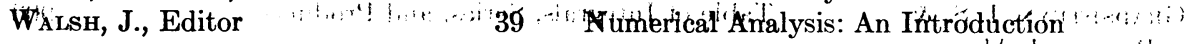

HALE, JACK K.
\& LASALLE;
b) $\|, \cdots, 11$
$: \cdots$

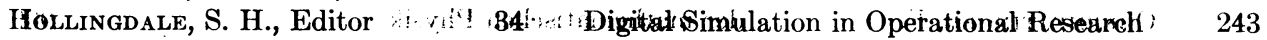

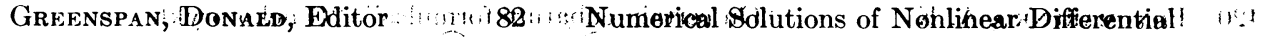

$\therefore:$ : Alnmw Equationd 896

Kruker, Melvin $\quad 893$

\& Korn, Granino A., Editors

Mond, Bertram, Editor $\quad 81 \quad$ Blanch Anniversary Volume $\quad 895$

$\begin{array}{llll}\text { SimonNard, Michel } & 48 & \text { Linear Programming } & 463\end{array}$

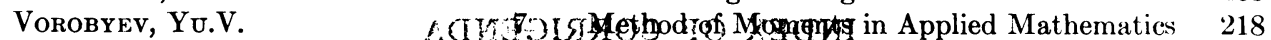

WALSH, J., Editor $\quad 39$ Numerical Analysis: An Introduction 4

13.15.1, Engineering Tables

Shipley, Herbert $\quad 13$ Standard Taples for Circular Curves ; , 227

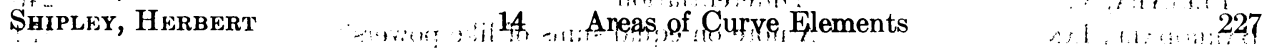

13.30 Economies and the Socral'Stiences

Simonnard, Michel _ 48 Linear Programming 463

ZUkнovitskiY, S.I." \& AvDEYEva, L. I.

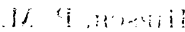

13.35 Information Theorysin A itomeata; Controd Theory, Dynamic Programming,

$1 \%$

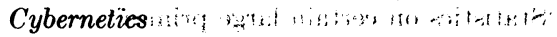

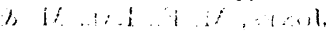

Cruon, R., Editor

73 Queuing Theory: Recent Developments drid wit

! $! \cdot$ Applications

689

Greenspan, Donald, Editor 82 Numerical Solutions of Nonlinear Differential in:

HÁte, JACK K. "Anstions 463

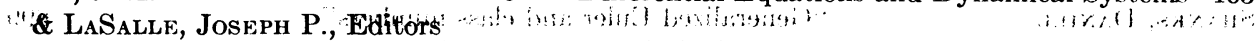

Hartmanis, J.

\& STEARNS, R. E.

Höllingdale, S. H., Editor Machines

235

KaUfmann, A.

34 Digital Simulation in Operational'Research

62 Graphs, Dynamic Programming, 'and 'Frte

Games

683

Klerer, Melvin

78 Digital Computer User's Handbook

893 \& Korn, Granino A., Editors earoy

13.40 Management Problems, Data Analysis and Processing , i yming

Hollingdale, S. H., Editor

Kterer, MeLvin \& Korn, Granino A., Editors
34 Digital!Simulation in, Operational Research in, 243

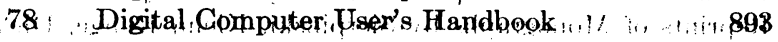




\section{INDEX OF TABLE ERRATA}

No.

Author

Title

Page

418 Abramowitz, Milton \&

Stegun, Irene A., Editors

421

423

419

Anema, A. S. \&

Miksa, F. L.

Handbook of Mathematical Functions with Formulas, Graphs, and Mathematical Tables

"Tables of primitive pythagorean triangles with equal perimeters"

Tables of Integral Transforms

Oberhettinger, F. \&

Tricomi, F. G.

INDEX OF CORRIGENDA

\section{Author}

Ballester, C. \&

Pereyra, V.

Barrodale, IAN

Bergman, Stefan \&

Chalmers, Bruce:

Fairweather, G. \&

Gourlay, A. R.

Hinsch, P. M.

Jones, M. F., LAL, M. \& Buundon, W. J. ,

\footnotetext{
Mangad, Moshe

Shanks, Danifl

Shanks, Danifl \&

Wrench, JoHN W., JR.

Shanks, Daniel \&

WRENCH, JoHN W., JR.
}

Title

Page

Supplement to Bickley's Table for Numerical Differentiation

"A note on equal sums of like powers"

"Procedure for conformal mapping of triply-connected domains"

"Some stable difference approximations to a fourth-order parabolic partial differential equation"

"Evaluation of orthogonal polynomials and relationship to evaluating multiple integrals"

"Statistics on certain large primes"

"Some limiting values and two error estimation procedures for power series approximations"

\section{INDEX OF NOTES}




\section{INDEX OF MICROFICHE SUPPLEMENTS}

Author

Covy, W. J.

Feuchter, C. A.

Osborn, D. \& Madey, R.

Patrerson, T. N. L.

Patrerson, T. N. L.

VARAH, J. M.

VARAH, J. M.

Weil, J., Murty, R. S. \& RAO, D. B.
Title

MOC Issue

Chebyshev Approximations for the Fresnel Integrals

Numerical Integration over a Sphere

The Incomplete Beta Function and its Ratio to the Complete Beta Function

On Some Gauss and Lobatto Based Integration Formulae

April

April

January

October

The Optimum Addition of Points to Quadrature Formulae

The Calculation of the Eigenvectors of a General Complex Matrix by Inverse Iteration

Rigorous Machine Bounds for the Eigensystem of a General Complex Matrix

Zeros of $J_{n}(\lambda) Y_{n}(\eta \lambda)-J_{n}(\eta \lambda) Y_{n}(\lambda)$ and $J_{n}^{\prime}(\lambda) Y_{n}^{\prime}(\eta \lambda)-J_{n}^{\prime}(\eta \lambda) Y_{n}^{\prime}(\lambda)$

October

October

October

January

\section{ADDENDUM}

Hunter, H. E., Kirk, I. B., Tables of Prolate Spheroidal Functions for $m=0$ SENIOR, T. B. A. \& WitThenberg, E. R. 